\title{
Scalability of photochemical reactions in continuous flow mode
}

\author{
Kian Donnelly ${ }^{1}$ (D) $\cdot$ Marcus Baumann ${ }^{1}$ (B) \\ Received: 9 March 2021 / Accepted: 25 March 2021 / Published online: 17 May 2021 \\ (C) The Author(s) 2021
}

\begin{abstract}
Continuous flow photochemistry as a field has witnessed an increasing popularity over the last decade in both academia and industry. Key drivers for this development are safety, practicality as well as the ability to rapidly access complex chemical structures. Continuous flow reactors, whether home-built or from commercial suppliers, additionally allow for creating valuable target compounds in a reproducible and automatable manner. Recent years have furthermore seen the advent of new energy efficient LED lamps that in combination with innovative reactor designs provide a powerful means to increasing both the practicality and productivity of modern photochemical flow reactors. In this review article we wish to highlight key achievements pertaining to the scalability of such continuous photochemical processes.
\end{abstract}

Keywords Flow synthesis $\cdot$ Photochemistry $\cdot$ Scalability $\cdot$ Throughput $\cdot$ Continuous processing

\section{Introduction}

Over the last decade continuous flow photochemistry has matured into a powerful field within synthetic organic chemistry. Key features that enable effective photochemical transformations in flow mode are the ability to uniformly irradiate solutions of substrates that are continuously pumped through narrow diameter tubing or microchannels [1-3]. High spatiotemporal control furthermore allows the effects from overirradiation to easily be minimised as in principle each molecule resides within the irradiated section of the flow reactor for the same amount of time, which can be controlled with high precision in flow mode [4]. Together with facile temperature control and various light sources being available (UV and visible), both commercial and home-built reactor systems render easy access to photochemical reactions. In addition, important advances in the field of photo-redox catalysis [5-7] mean that synthetic chemists can choose between directly irradiated and modern photo-catalysed reactions that provide mild and selective means to manipulate and functionalise compounds of ever-increasing complexity. One important argument frequently put forward by practitioners in favour of

Marcus Baumann

marcus.baumann@ucd.ie

1 School of Chemistry, Science Centre South, University College Dublin, D04 N2E2, Dublin, Ireland continuous flow processing over batch mode operation of photochemical processes concerns their scalability. Whilst scale-up in batch mode is primarily actualised through an increase in vessel size, analogous flow processes can be scaledup through prolonged process times as well as specific design features that render a variety of reactions more productive [8].

A crucial parameter to be considered during any photochemical reaction is that of light penetration. Light is absorbed by the reaction medium, and this light attenuation is dependent on the irradiated reactor size as dictated by the BeerLambert law (Fig. 1). As the size of a reactor increases so too does the path length (distance from the light source), resulting in non-uniform irradiation. This typically is one of the key issues for scaling up photochemical reactions in batch mode, however the analogous flow processes overcome this issue through miniaturisation strategies in order to shorten the path length.

In 2014 Booker-Milburn and co-workers published an important account [9] comparing the scalability and efficiency of flow and batch based photoreactions and concluded at that stage that there is very little difference when key parameters are kept the same. In their work a dozen transformations including $[2+2]$-cycloadditions between maleimides and alkenes or alkynes (intra- and intermolecular), a Paterno-Büchi cycloaddition, a di- $\pi$-methane rearrangement as well as several radical-based processes were evaluated. Productivities of several decagrams per day were typically achieved for these optimised processes. At this stage flow reactor configurations 


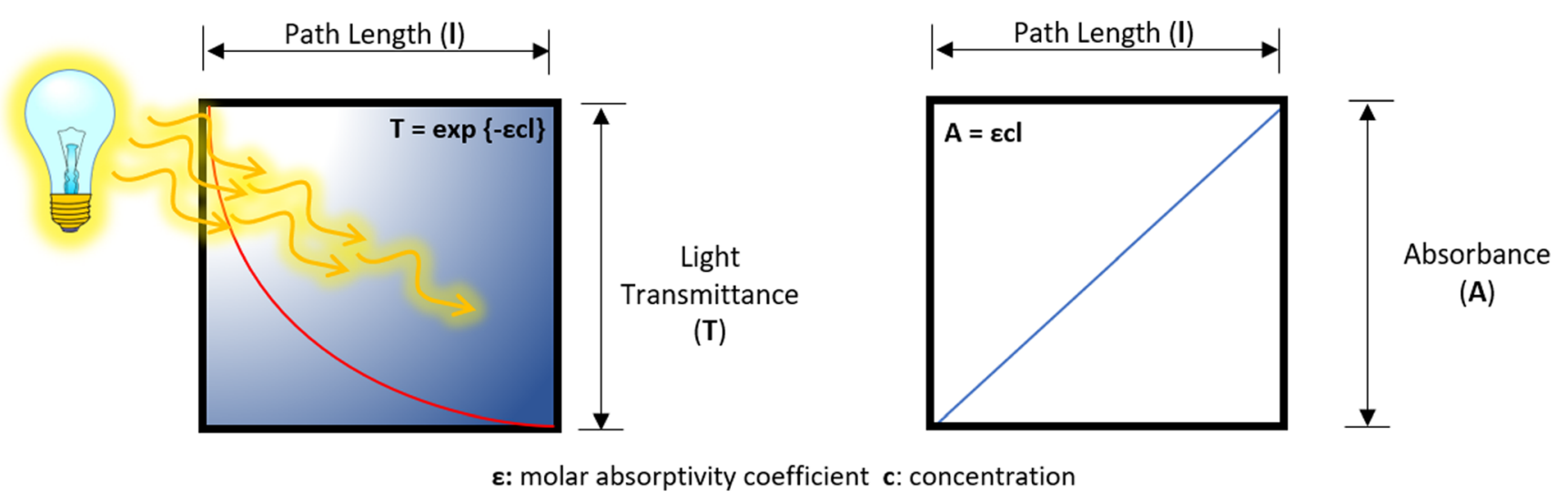

Fig. 1 Attenuation of light through a reactor vessel expressed via transmittance and absorbance

were commonly based on FEP-coil reactors (single and multiple layers) in combination with UV-C or medium-pressure Hg-lamps whereas immersion type reactors using the same lamps were used in batch mode. Despite the slightly different set-ups and irradiation profiles, the conclusion of equal performance for batch and flow processing could be drawn. It should be noted that this comparison focused on reactions in which substrates are directly irradiated, and therefore the conclusions may not extend to more complex reactions involving photocatalysts.

More recently, a variety of new photoreactors designed for continuous processing have been developed. These include commercial systems such as the Vapourtec UV150 reactor and its high-power LED extension [10-14], the Corning photoreactor system [15-17], the Uniqsis PhotoSyn [18, 19], or the Firefly system from Cambridge Reactor Design [20]. Furthermore, studies employing a modified rotary reactor (coined PhotoVap) [21], a vortex reactor [22, 23] or oscillatory flow reactors for heterogeneous reactions [24, 25] have been reported. It is not surprising that the design of such newer systems and the implementation of chemical engineering knowledge increases the performance and therefore scalability of the resulting applications. In addition, a growing body of today's literature is based on photosensitised transformations that oftentimes exploit visible rather than UV light.

In view of these manifold developments, we wish to provide an update on recent contributions by discussing relevant studies and highlight new knowledge pertaining to the scalability of flow-based photochemistry. Specifically, we will discuss scalability as a function of the equipment used, the underlying photo-transformation as well as the complexity of substrates and products involved. We hope that this review allows interested readers to find not only interesting applications of continuous photochemical reactions, but moreover appreciate the complex interplay of different factors that determine success and ultimately scalability in these light-driven processes.

\section{Discussion}

This first section will highlight several modern as well as classical photochemical transformations that are difficult to execute and scale in batch mode. Transferring the initial batch reaction into continuous flow mode thus provides a means of generating gram quantities of desired target products in a time and cost-effective manner. These laboratory scale examples therefore demonstrate approaches for increasing the productivity and practicality of various light-driven reactions. Simple flow setups are thereby utilised to satisfy the demand of materials of interest whose scaled preparation can be advanced further by scale-out and numbering up strategies. Subsequent sections will then expand on this theme by introducing setups and concepts enabling productivities of several kilograms per day. The productivities thus reached will in many cases be sufficient to meet the current demand for specific branches within industry (pharmaceutical, fragrances and fine chemicals), although it is understood that these are subject to further changes over time.

\section{Laboratory scale $(<100$ g/day)}

Photoredox catalysis has experienced a surge in interest in recent years [26-31], in part, due to the advancement of equipment such as LED lamps used for photochemical reactions. Transition metal-mediated photoredox catalysis is generally characterised by two coupled catalytic cycles. Firstly, a cycle activating the photocatalyst, which then turns over the substrate in the second catalytic cycle. Photoredox catalysis can be exploited for a wide range of reactions including various carbon-carbon bond forming reactions [29-31]. Recently, the MacMillan group reported a batch method for the photoredox catalysed formation of $\mathrm{C}_{\mathrm{sp}}{ }^{3}-\mathrm{C}_{\mathrm{sp}}{ }^{2}$ bonds [32]. This was performed by coupling aryl halides to alkyl halides in the presence of an iridium-nickel co-catalytic system and a silyl radical precursor (Scheme 1). The methodology proved to be robust giving the desired products in good yields, however, the reaction was only carried out on small scale. 
Scheme 1 Photochemical $\mathrm{C}_{\mathrm{sp}}{ }^{3}$ $\mathrm{C}_{\mathrm{sp}}{ }^{2}$ coupling<smiles>COC(=O)c1ccc(Br)cc1</smiles>

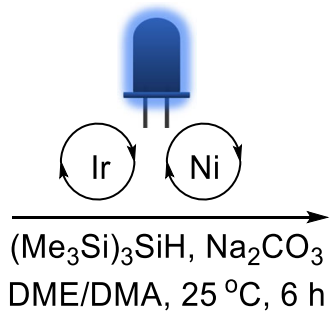

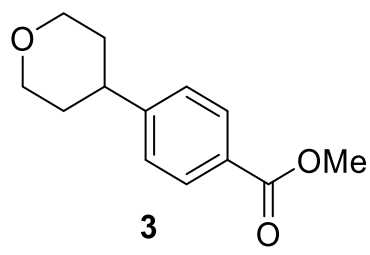

In order to utilise this reactivity in a scalable manner, the Jensen group developed a continuous stirred-tank reactor (CSTR) system for carrying out photochemical reactions [33]. The reaction reported by MacMillan required the presence of an insoluble inorganic base, which typically is incompatible with carrying out reactions in a continuous manner due to reactor blocking and fouling. However, the use of a CSTR could mitigate this issue through careful reactor design. Previously, the Jensen group had reported the design of a CSTR cascade used for heterogenous reactions [34], which was adapted for this photochemical reaction.

The reactor consisted of five single chambers (total volume $5.3 \mathrm{~mL}$ ), connected by a drilled channel, which could be irradiated through a glass window (Fig. 2). Mixing was achieved using magnetic stir bars which could be operated using a conventional magnetic stirring plate. Using this setup, in combination with a slurry pump, the heterogeneous mixture could be administered to the reactor while maintaining an inert atmosphere.

Under these conditions facile direct transfer of the previous batch method to continuous flow was possible, eliminating the need for re-optimisation to incorporate a soluble organic base. The flow system was then used to synthesise compound $\mathbf{3}$ on a gram scale in $13 \mathrm{~h}(77 \mathrm{mg} / \mathrm{h})$ with similar yields to the batch process (77\% in flow, $80 \%$ in batch).

While the application of flow chemistry to this reaction did not offer an improvement in yield, as is sometimes the case, it

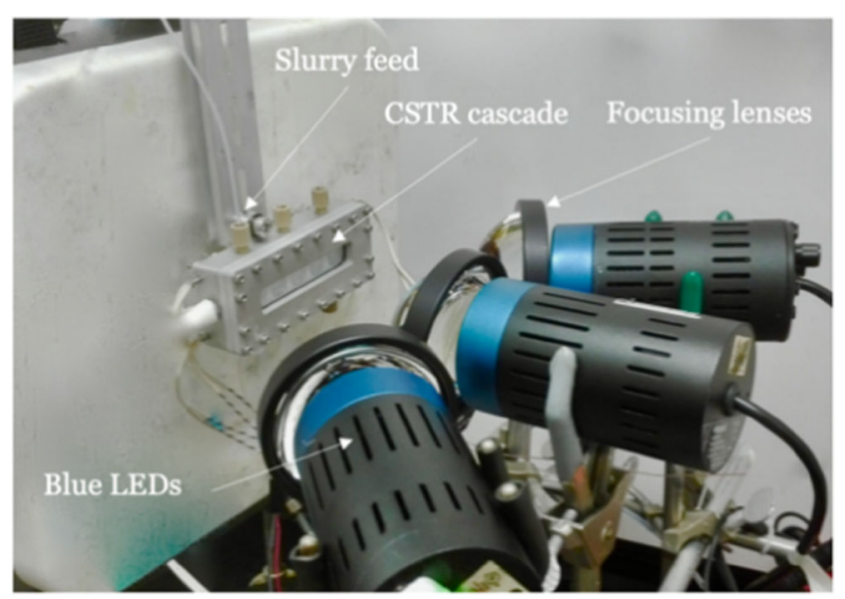

Fig. 2 Image of CSTR setup. Reprinted with permission from [33]. Copyright (2019) American Chemical Society offered significantly shorter reaction times (30-min residence time vs $15 \mathrm{~h}$ in batch) and higher productivity than a batch reactor of similar size. Based on the reactor design this productivity could be further improved by a relatively straightforward increase in reactor size.

The applicability of continuous flow to scaling up photoredox processes has also been demonstrated by the Collins group [35], who reported the synthesis of alkynyl sulfides 6 via C(sp)-S bond formation (Scheme 2). The coupling of alkynyl bromides 5 and thiols $\mathbf{4}$ was performed using a 4CzIPN (an organic carbazole-based sensitiser [36]) and nickel co-catalysed system. While the reaction could be carried out successfully in batch on a small scale $(0.24 \mathrm{mmol})$ with excellent yields (92\%) the long reaction time of $4 \mathrm{~h}$ was unfavourable. The use of a continuous flow system allowed for significantly shorter reaction times of $30 \mathrm{~min}$ while maintaining high yields.

The simple continuous flow setup consisted of a syringe pump and a homemade PFA coil reactor which surrounded a portion of blue LED ribbon $\left(\lambda_{\max }=465 \mathrm{~nm}\right.$, Fig. 3). Scale-up of the photochemical reaction in batch proved challenging with a significantly reduced yield of $26 \%$ being obtained when carried out on a gram-scale $(5 \mathrm{mmol})$. Furthermore, the low concentration required for the reaction $(50 \mathrm{mM})$ would make further scale-up in batch impractical due to the requirement for increasingly large reactor vessels. In contrast, a scale-out of the reaction in continuous flow yielded over $1.2 \mathrm{~g}$ of product with significantly improved productivity over batch $(1.16 \mathrm{mmol} / \mathrm{h}$ vs $0.33 \mathrm{mmol} / \mathrm{h})$.

An issue that commonly arises during photochemical reactions is that of over-irradiation of either substrate or the resulting reaction product. This can lead to a variety of side reactions occurring and is typically unavoidable in batch. The advantage offered by spatiotemporal processing in continuous flow typically eliminates this issue allowing sensitive reactions to be carried out on a larger scale.

The improvement in flow technology in recent years has provided chemists with the opportunity to revisit chemistry that previously had not been scalable. One such example is the photo-electrocyclization of 2-pyrone derivatives which was first reported by Corey in 1964 [37]. The resulting cyclobutene lactone product $\mathbf{8}$ is a synthetically versatile chemical building block due to its high ring strain [38-41]. Recently, the Kappe group investigated the applicability of 
Scheme 2 Continuous flow synthesis of alkynyl sulfides

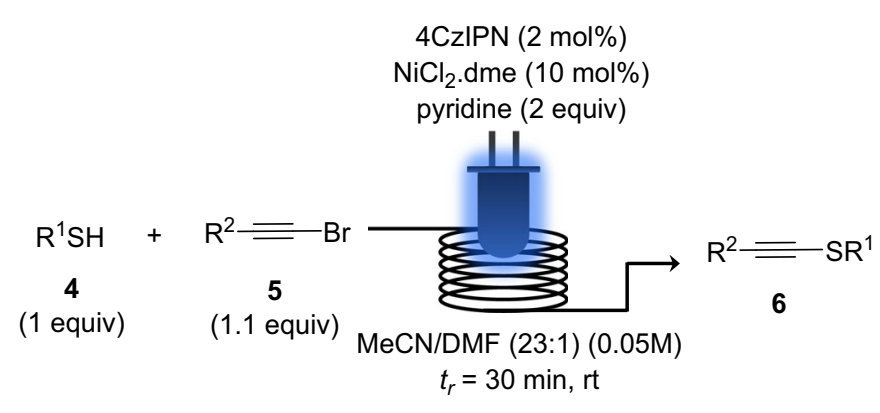

flow chemistry to improving the scalability of this reaction [42]. Careful optimisation of reaction conditions found the correct selection of light source to be of critical importance, with an improvement in yield being observed using a commercially available medium pressure mercury lamp (with the appropriate light filter) when compared to a weaker $8 \mathrm{~W} \mathrm{UV}$ lamp emitting at $303 \mathrm{~nm}$. Use of the correct light source allowed the researchers to synthesise the desired cyclobutene lactone $\mathbf{8}$ in high yields (> 95\%) with significantly reduced reaction times when compared to batch (20-min residence time vs $24 \mathrm{~h}$ in batch). To scale up the flow process the commercially available Vapourtec UV-150 reactor $(10 \mathrm{~mL})$ was used (Scheme 3). While attempting to scale-out the process by running the reactor for $8 \mathrm{~h}$, significant reactor fouling was observed due to the formation of an insoluble polymeric material, as is the case in batch. This was resolved by decreasing the reaction temperature from $50{ }^{\circ} \mathrm{C}$ to $10{ }^{\circ} \mathrm{C}$, however, a conversion decrease of $2.3 \%$ per hour was still observed. Despite this, the continuous flow setup achieved a productivity of $144 \mathrm{mg} / \mathrm{h}(\sim 3.5 \mathrm{~g} /$ day $)$ over an 8-h run, significantly higher than previously reported batch reactions $(14-21 \mathrm{mg} /$ h, 336- $504 \mathrm{mg} /$ day) [41].

Photochemically induced cyclisation reactions have been applied to the synthesis of a range of natural product derivatives. $[43,44]$ Typically, such synthetic projects do not require kilograms of product but the isolation of anything more than hundreds of milligrams can prove challenging in batch. This results in the chemist having to carry out multiple reactions in

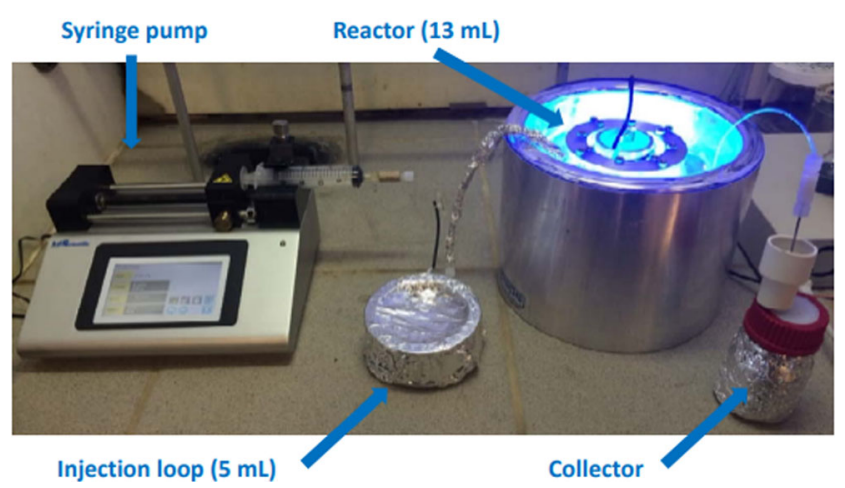

Fig. 3 Image of continuous flow setup (reproduced with permission from [35]) order to isolate multiple grams of the desired material. As an alternative, researchers have increasingly begun to utilise continuous flow as part of their synthetic strategy. One recent example was published by the Beeler group who developed the gram scale photochemical synthesis of aglain analogues, a precursor to rocaglate natural product analogues. [45] A common strategy for the synthesis of aglain $\mathbf{1 2}$ is the photochemical [3 +2]-cycloaddition of 3-hydroxyflavone $\mathbf{1 0}$ and methyl trans-cinnamate (11). [46, 47] Previous reports in batch typically provide $<150 \mathrm{mg}$ of product per reaction in $40-50 \%$ yield with long reaction times of up to $12 \mathrm{~h}$. [47]

Initial optimisation was carried out using a reactor consisting of a $150 \mathrm{~W}$ metal-halide lamp $(>300 \mathrm{~nm})$ placed at the centre of a FEP coil reactor $(3.5 \mathrm{~mL})$. Recirculating glycol was used to cool the inner chamber of the reactor. Under the optimised conditions the desired product $\mathbf{1 2}$ could be synthesised with a residence time of 30 min giving a throughput of $\sim 180 \mathrm{mg} / \mathrm{h}$ compared to the batch throughput of $\sim 11 \mathrm{mg} / \mathrm{h}$. To further scale up the reaction, the volume of the photochemical reactor was increased to $12.5 \mathrm{~mL}$ (Scheme 4). Numbering up this larger reactor to three gave a total volume of $37.5 \mathrm{~mL}$, which, with a slight increase in residence time to $35 \mathrm{~min}$, further increased the throughput to $1.92 \mathrm{~g} / \mathrm{h}$. This strategy exemplifies the ease at which scalability of a continuous flow process can be achieved when compared to its batch counterpart.

Conformationally restricted three dimensional molecules are becoming increasingly important scaffolds for medicinal chemistry applications $[48,49]$. One method by which these

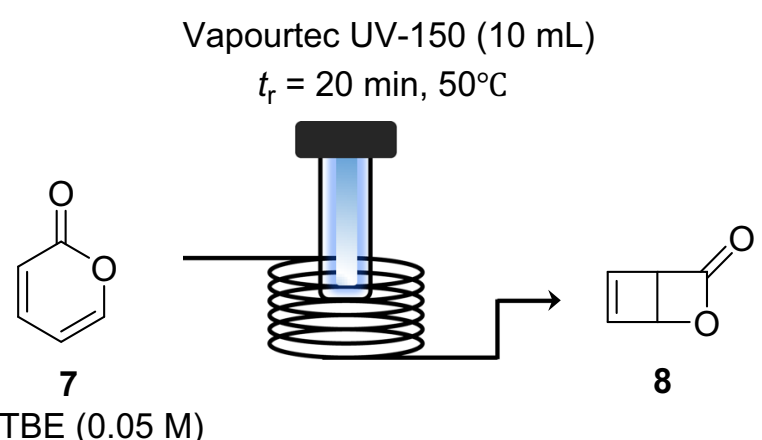

in MTBE $(0.05 \mathrm{M})$

Scheme 3 Photochemical synthesis of 2-oxabicyclo[2.2.0]hex-5-en-3one 8 
Scheme 4 Photochemcial synthesis of aglain $\mathbf{1 2}$
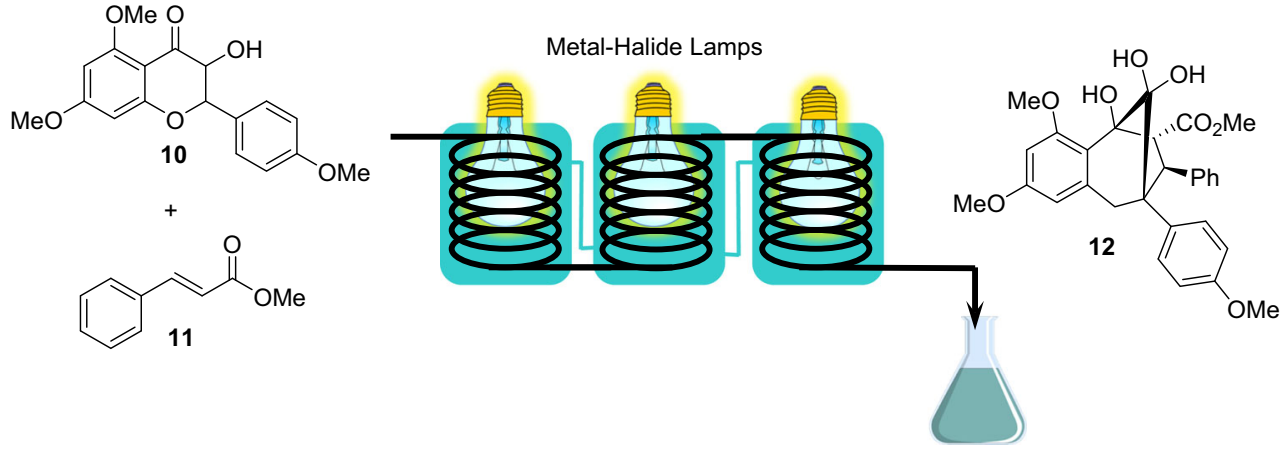

structures can be accessed is through photochemical intra- or intermolecular cycloaddition reactions [50]. This strategy was used by the Rutjes group for the scalable synthesis of cyclic aminoketones in continuous flow mode (Scheme 5) [51].

For optimisation purposes reactions were carried out on a small scale using a 'home-made' reactor. This small-scale reactor consisted of FEP tubing $(11.6 \mathrm{~mL})$ wrapped around a condenser, which was then irradiated using a commercially available Rayonet RMR-600 photochemical reactor. Low substrate concentrations $(30-40 \mathrm{mM})$ were found to provide excellent yields when irradiated at $254 \mathrm{~nm}$ for $30-40 \mathrm{~min}$ depending on the substrate.

Using these optimised conditions, a throughput of $\sim 5.6 \mathrm{~g} /$ day was achieved. However, this scale was insufficient for the required synthesis of these compounds. Further scale-up was therefore achieved through design of a larger reactor (Fig. 4). This system consisted of FEP tubing (volume: $105 \mathrm{~mL}$ ) wrapped around a commercially available UV-C lamp. Cooling of the reactor was achieved using an external stream of water, and the system was encased with a metal jacket to protect the user from harmful UV radiation. Using this high throughput setup $10 \mathrm{~g}$ of compounds $\mathbf{1 4}$ and $\mathbf{1 6}$ could be synthesised in 4.8 and $7.3 \mathrm{~h}$, respectively. This equated to a much-improved throughput of $\sim 30-50 \mathrm{~g} /$ day, achieved through a straightforward increase in reactor size.

While the above examples demonstrate how flow chemistry can be used to scale photochemical reactions, generally most reactions must be reoptimized to transfer from batch to flow. This can typically result in lost time trying to find the optimal flow rate to achieve sufficiently high yields. Recently the Booker-Milburn group undertook a study in an attempt to rectify this issue [52]. It was reported that the transfer of optimised batch conditions to flow could be simplified by calculating the flow rate based on batch parameters. This would help reduce development time as reactions can be optimised more rapidly in batch due to the ability to easily monitor them in real time, without the need for additional expensive equipment. To verify this hypothesis, various reactions were transferred from batch to continuous flow. Among these was the photorearrangement of $N$-substituted succinimides 17 to keto-caprolactams 20 (Scheme 6) [53, 54].

While well-known, this reaction proves difficult to scale due to alternative competing reaction pathways leading to the formation of insoluble side-products, particularly at higher concentrations. The flow rate in UV photochemistry is of prime importance due to the ease at which reaction mixtures can be
Scheme 5 Photochemical synthesis of conformationally strained scaffolds<smiles>C=CCN(C(=O)OCc1ccccc1)C1=CC(=O)CCC1</smiles>

$13 a$<smiles>C=CCCN(C1=CC(=O)CCC1)C(C)(C)C</smiles>

$13 b$

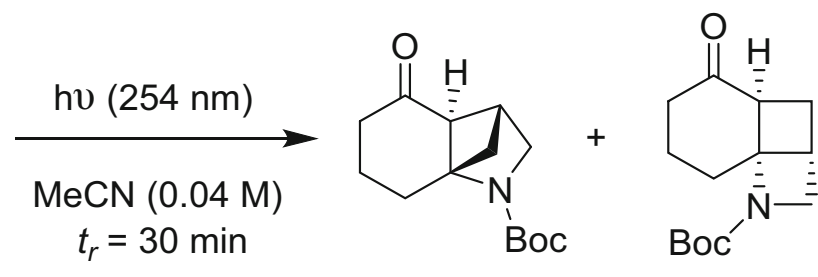

14

$96 \%(4: 1)$

15

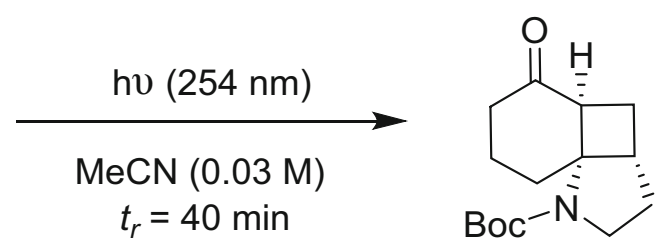

$16(95 \%)$ 


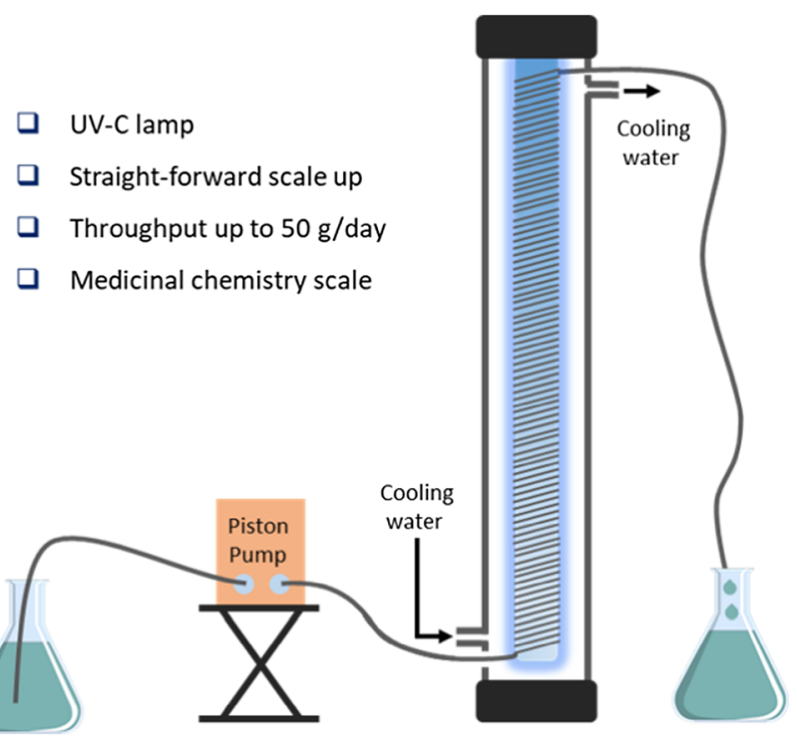

Fig. 4 Medicinal chemistry scale continuous flow setup

over-irradiated, leading to diminished yields and side-product formation. Based on previous reports stating that FEP flow reactors have similar productivities per Watt to batch [9], the optimal flow rate could be calculated based on the power of the flow setup. To test this, a reactor consisting of FEP tubing wrapped around $3 \times 36 \mathrm{~W}$ UV-C lamps was used. Based on the increase in power compared to batch $(18 \mathrm{~W})$ a flow rate of $7.5 \mathrm{~mL} \mathrm{~min}^{-1}$ was calculated. Through re-optimisation of the flow procedure a flow rate of $8 \mathrm{~mL} \mathrm{~min}^{-1}$ was found to be ideal, closely matching the calculated value. While no improvement in the yield of $\mathbf{2 0}$ was observed, the productivity of the reaction was significantly improved to $96 \mathrm{~g} /$ day compared to the previously reported $2.2 \mathrm{~g}$ /day in batch [54]. While using a continuous flow setup does not lead to an increase in reaction efficiency, it does allow for facile scale up due to the potential for increased reactor power as a function of the presence of multiple lamps. However, it should be noted that this conclusion may only be drawn for the reaction included in the survey, as photocatalysed pathways may display different behaviour due to their increased complexity.

\section{Pilot scale $(100+$ g/day $)$}

While the above examples demonstrate the advantages of continuous flow in scaling of reactions to the decagram scale, various examples of equivalent batch reactions on this scale exist [9]. However, as reaction scale approaches the order of hundreds of grams the use of a batch setup becomes increasingly impractical due to the requirement for significantly larger equipment as well as limitations regarding heat transfer. Low concentrations and high lamp powers required for successful photochemical reactions add to these complications.

Carbon-carbon bond formation reactions are of extreme importance in a chemist's toolbox for which a range of methodologies exist [31, 55, 56]. Recently, Alcázar and co-workers reported the visible-light induced Negishi reaction for $\mathrm{C}\left(\mathrm{sp}^{3}\right)$ $\mathrm{C}\left(\mathrm{sp}^{2}\right)$ cross couplings (Scheme 7) [57, 58]. This two-step reaction involves firstly the formation of an organozinc reagent $\mathbf{2 2}$, which is then coupled with an aryl bromide $\mathbf{2 3}$ under photochemical conditions in the presence of a nickel catalyst. This photochemical methodology presented a broader scope than the traditional nickel-catalysed Negishi reaction and was scaled to achieve a throughput of $800 \mathrm{mg} / \mathrm{h}$ using a commercially available Vapourtec reactor. While useful for screening purposes, a larger scale was required for 'pilot' scale. Good yields had been reported using the Vapourtec system, however, its scalability was limited by its fixed reactor volume of $10 \mathrm{~mL}$. To overcome this limitation, the authors opted to use a larger Corning G1 reactor $(40 \mathrm{~mL})$ for the photochemical process (Fig. 5).

The continuous flow setup consisted of a column filled with powdered zinc, to generate the required organozinc reagent (22) in a continuous process that was monitored using in-line NMR, which was combined with a stream of aryl bromide and nickel catalyst prior to entering the photoreactor. The use of a high-powered LED lamp (100 W, $405 \mathrm{~nm})$ provided the desired product in excellent yield matching that of the previous Vapourtec example. Optimisation of the reaction found that a slightly shorter residence time could be utilised further improving the throughput to $5.6 \mathrm{~g} / \mathrm{h}$. Use of the larger reactor offered a sevenfold increase in throughput (134 g/day vs $19 \mathrm{~g}$ /day) when compared to the smaller Vapourtec reactor despite only a fourfold increase in volume (Scheme 7). It is worth noting that this non-linear increase may be attributed to the increased light power-to-surface area offered by using glass plate reactors in place of the traditional FEP tubing setup.

The incorporation of fluorine into organic molecules is highly relevant to the pharmaceutical industry due to their
Scheme 6 Photorearrangement of $N$-substituted succinimide<smiles>[R]CCN1C(=O)CCC1=O</smiles>

17

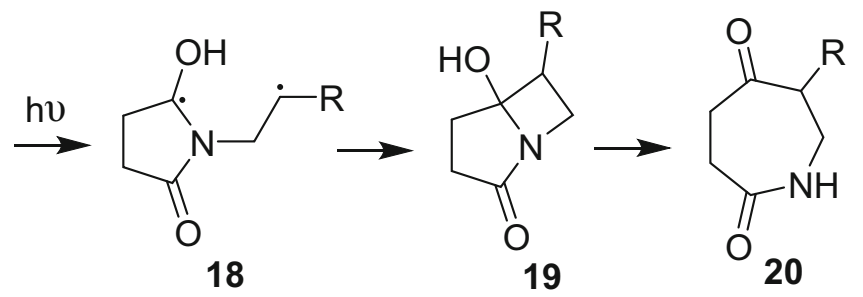




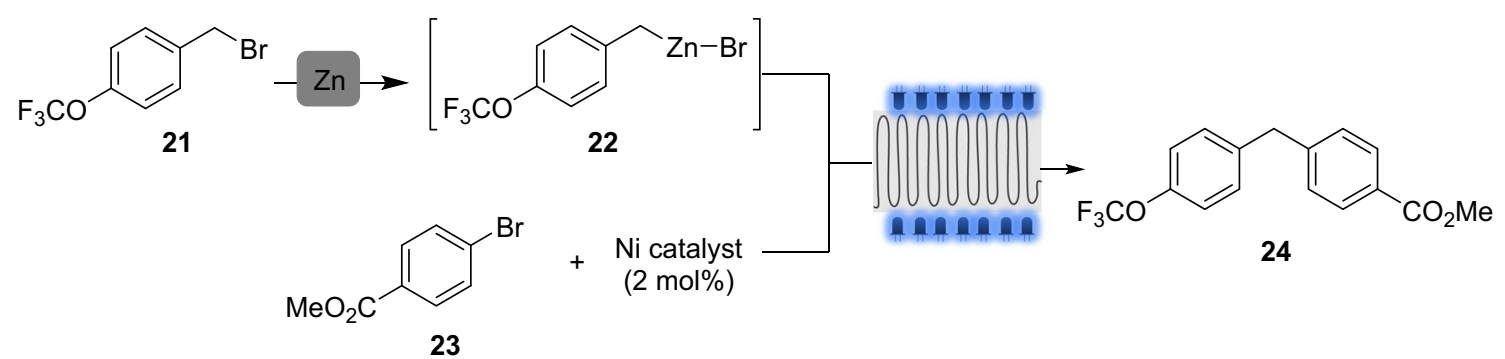

$\begin{array}{lll} & \text { Vapourtec } & \text { Corning G1 } \\ \text { Yield }(\%) & 93 & 93 \\ \text { Time (min) } & 20 & 15 \\ \text { Reactor size }(\mathrm{mL}) & 10 & 40 \\ \text { Throughput }(\mathrm{g} / \mathrm{h}) & 0.8 & 5.6\end{array}$

Scheme 7 Visible-light induced Negishi-coupling reactions

potential to alter biological properties of molecules $[59,60]$. However, there remains a lack of scalable methodology to achieve this. This limitation arises, in part, due to the cost, both monetary and environmental, associated with typical fluorinating and trifluoromethylating reagents. Recently, the Stephenson group developed a photochemical method for incorporating trifluoromethyl groups into various arenes and heteroarenes $[61,62]$. This reaction involved the photochemical decarboxylation of trifluoroacetic anhydride (26) facilitated by pyridine $N$-oxide in the presence of a ruthenium catalyst (Scheme 8). Blue LEDs (13 W) were found to be a sufficiently powerful light source, and the desired trifluoromethylated products could be obtained from a range of substrates in good yields. Despite promising results, this methodology had not previously been utilised for scales larger than $20 \mathrm{~g}$.

In order to further scale up this reaction, the Stephenson group opted for a continuous flow setup based on a coil reactor design [63]. The reactor consisted of blue LED lights contained within a glass beaker with a single layer of PFA tubing wrapped around the outside. The total reactor volume measured approximately $150 \mathrm{~mL}$ and the assembly was housed within a steel casing. Cooling of the reactor was achieved through filling of both cavities with water. This setup fulfilled the predetermined requirements of low cost and having a small footprint (Fig. 6). Using this 'home-made' reactor the trifluoromethylation of pyrrole $\mathbf{2 5}$ was carried out on the kilogram scale. Running the reactor continuously for $48 \mathrm{~h}$ provided $0.95 \mathrm{~kg}$ of product 27 , equating to $20 \mathrm{~g} / \mathrm{h}$. The equivalent reaction in batch would prove impractical due to the size of the reaction vessel required, in addition to the size and power of the light source.

Many examples of continuous flow photochemical reactors in the literature consist of tubing wrapped around a light source $[1,3,64]$. However, various other creative solutions to benefit from the advantages of continuous flow have been reported [65]. In 2016 the George group presented a photochemical reactor comprised of a converted rotary evaporator which was coined the PhotoVap [66]. This setup could be run
Fig. 5 Continuous flow setup: 1. G1-photoreactor; 2. Zinc column; 3. Reagents; 4. Mass flow controller; 5. Back pressure regulator; 6 . Collection vessel; 7 . Zinc activation waste; 8 . Pressure and temperature monitor; 9 .

Needle valve; 10 . Pressure sensor. (Reproduced with permission from [58])

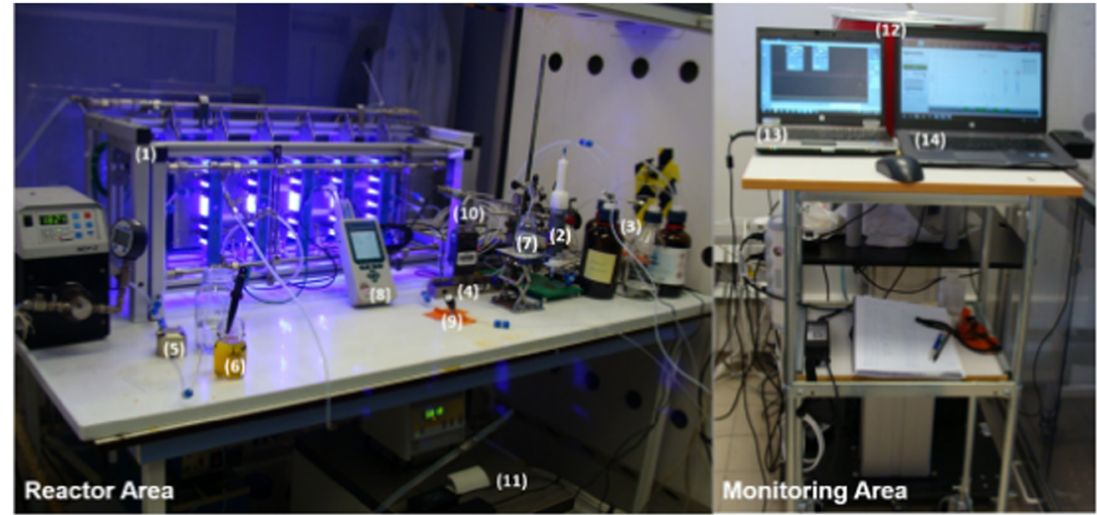


Scheme 8 Continuous flow photochemical trifluoromethylation

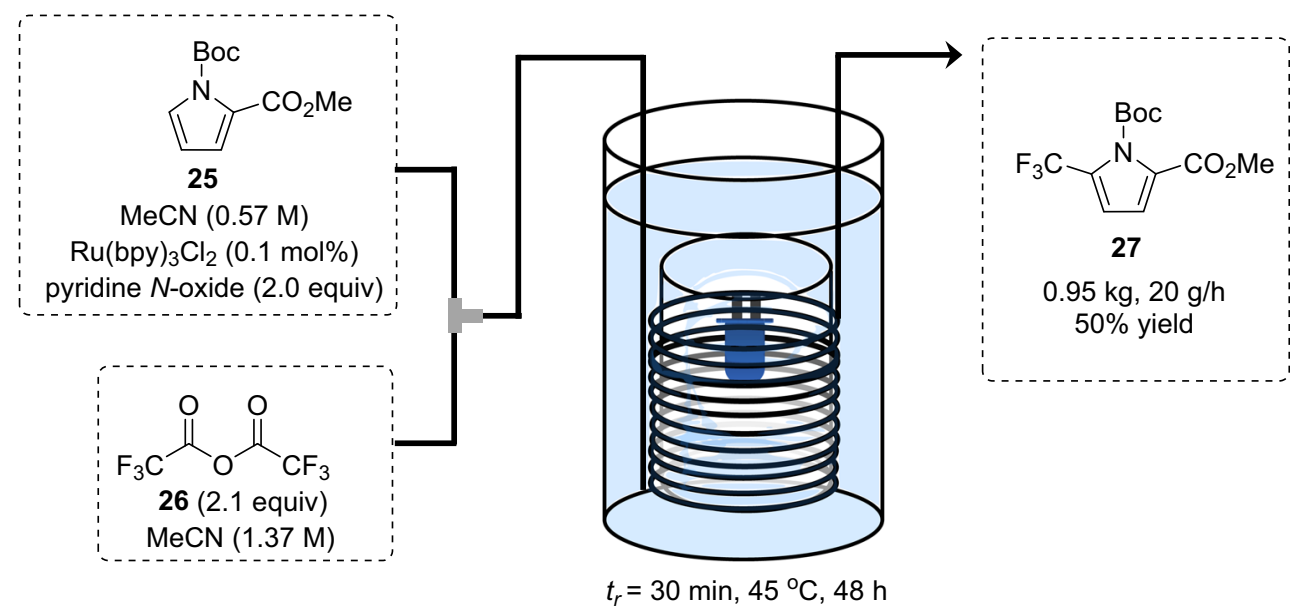

in a semi-continuous manner and required little modification to the conventional, widely available laboratory rotary evaporator (Fig. 7).

The reactor consisted of two thin PTFE tubes which were threaded through the neck of a rotating flask, which were used to dose starting material and subsequently withdraw product. The flask was surrounded by LEDs with temperature control achieved using the rotary evaporator bath. Dosing of reagents and rotation speed were controlled using an external computer. By rotating the flask, a thin film of reaction mixture on the vessel wall was generated, which provided more efficient radiation than might be observed in batch mode. Additionally, the scale of the reaction could be adjusted by increasing the size of the reaction flask up to $3 \mathrm{~L}$ (other rotary evaporator models can facilitate larger flasks).

In order to demonstrate the scalability of this semicontinuous approach the photo-oxidation of $\alpha$-terpinene $\mathbf{2 8}$ to ascaridole 29 was selected (Scheme 9) [67]. This reaction utilises Rose Bengal as a photosensitiser, which in combination with light can generate singlet oxygen from either ambient air, or a stream of oxygen gas [68]. This highly reactive oxygen species then efficiently oxidises $\alpha$-terpene to the desired product 29. Oxygen gas was bubbled through the reaction mixture, by passing an additional PTFE tube into the flask, and the mixture was irradiated using $1000 \mathrm{~lm}$ white LEDs. To run the reaction semi-continuously, a $50 \mathrm{~mL}$ portion of substrate feed was administered and irradiated in the rotating flask until reaction completion was reached. Rotation was then ceased, and the resulting product was removed before repeating the cycle. Using a $3 \mathrm{~L}$ flask, a productivity of $1 \mathrm{mmol} / \mathrm{min}$ (which extrapolates to approximately $10 \mathrm{~g} / \mathrm{h}$, $240 \mathrm{~g} /$ day) could be achieved. While there are various scalable examples of this specific reaction in the literature, this reaction design presents a potentially versatile alternative to plug flow reactors for chemistry with short reaction times.

$[2+2]$-Cycloadditions remain one of the most common methods for accessing cyclobutanes [69]. Most of these reactions are carried out under photochemical conditions, in some cases in the presence of a photosensitiser. The Kappe group recently reported a scalable method for the photochemical $[2+2]$-cycloaddition of ethylene to cyclic anhydrides [70]. LEDs offer the advantage of being more efficient and generating less heat than their medium- or low-pressure mercury lamp counterparts. Additionally, they can be used to emit specific near-monochromatic wavelengths, however they generally are limited to longer wavelengths $(>350 \mathrm{~nm})$. Typically, the use of longer wavelengths requires the presence of a photosensitiser for certain reactions to facilitate the energy
Fig. 6 Kilogram-scale continuous flow setup (reproduced with permission from [61])
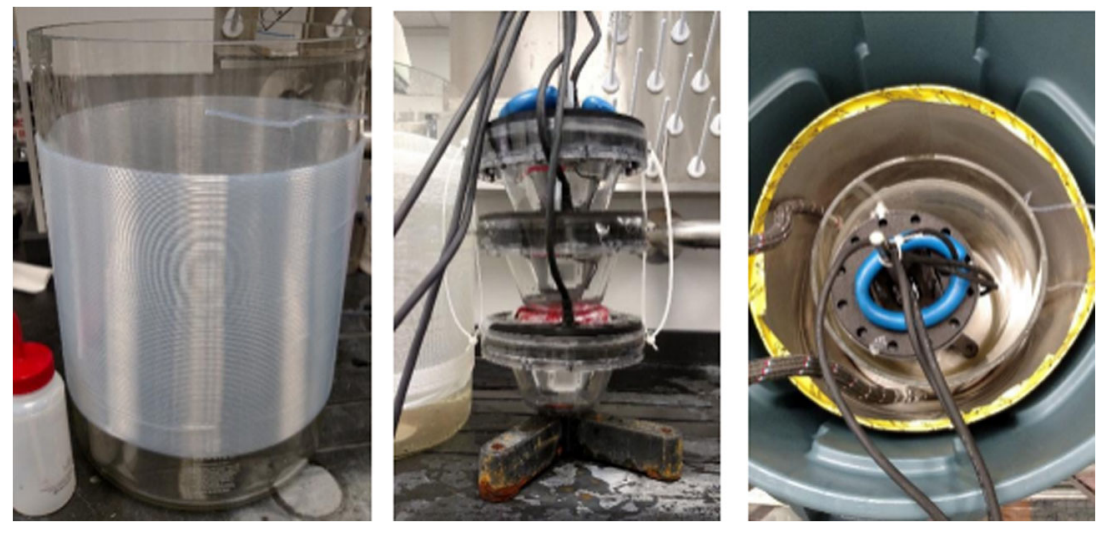


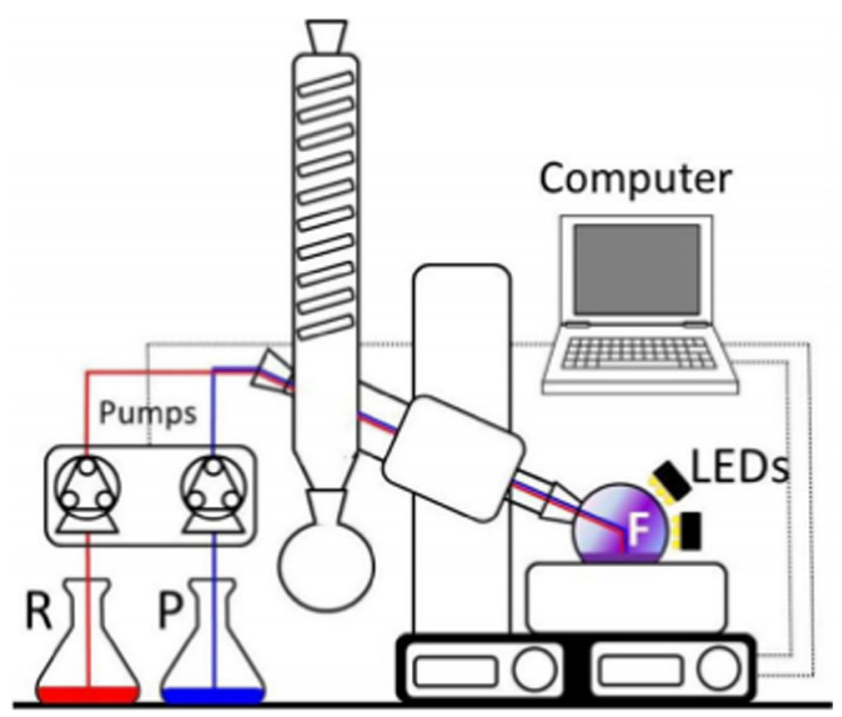

Fig. 7 'PhotoVap' setup. Reprinted with permission from [66] . Copyright (2016) American Chemical Society

transfer. This study by the Kappe group highlighted the importance of the selection of the correct photosensitiser and found thioxanthone (31) to be ideal for the reaction between citraconic anhydride (30) and ethylene to produce the corresponding cyclobutane 32 (Scheme 10).

The fact that ethylene is present as a gas makes carrying out this reaction non-trivial, in particular in batch, due to the associated hazards and complications of using a liquid-gas biphasic mixture. Initial experiments and optimisation were carried out using a commercially available plate reactor $(2.77 \mathrm{~mL})$, which was irradiated using two LED panels (375 nm). Starting anhydride $\mathbf{3 0}$ and photosensitiser $\mathbf{3 1}$ were dosed using integrated pumps and were combined with ethylene, with gas flow being regulated by a mass flow controller. A back pressure of 12 bar was applied to ensure a homogenous system, maximising contact between the substrates. Optimisation of the reaction found a residence time of $5.2 \mathrm{~min}$ to be sufficient for full conversion, corresponding to a productivity of $2.1 \mathrm{~g} / \mathrm{h}(\sim 58 \mathrm{~g} /$ day $)$. To demonstrate the scalability of this reaction, a switch to the Corning G1 photoreactor was made. This consisted of five $2.77 \mathrm{~mL}$ plate reactors connected in series giving a total volume of $13.85 \mathrm{~mL}$ (Fig. 8). Adjustment of the flow rate to maintain the constant $\sim 5.5 \mathrm{~min}$ residence time provided a productivity of $\sim 10 \mathrm{~g} / \mathrm{h}$<smiles>CC1=CC=C(C(C)C)CC1</smiles>

28

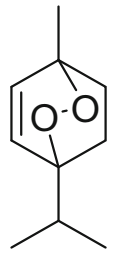

29
Scheme 9 Photooxygenation of $\alpha$-terpinene
( $\sim 240 \mathrm{~g} /$ day), with a 10 -h run yielding $101 \mathrm{~g}$ of $\mathbf{3 2}$. While the photochemical reaction may be feasible in batch, the use of ethylene gas on this scale would be unfavourable due to the potential safety risks, which are mitigated in continuous flow [71].

\section{Production scale (> $1 \mathrm{~kg} /$ day)}

While there are various examples exemplifying the advantages of continuous flow photochemistry for scale up, the transfer of these reactions from the laboratory scale to production scale is non-trivial. The previously discussed studies are generally limited by the volume of the reactor utilised, however, an increase in volume by increasing tubing diameter may alter the fluid dynamics of a reaction whilst also increasing the path length of the incoming light. Therefore, additional reaction optimisation may be required when attempting to perform continuous photochemical reactions at the kilogram scale. However, it should be noted that these differences in fluid dynamics observed during scale up are significantly smaller than those observed in batch.

With the scalability of the synthesis of cyclobutanes from ethylene being demonstrated by the Kappe group on the hundred-gram scale [70], no attempts had been reported of performing this reaction on manufacturing scale. Recently, Beaver, Zhang and co-workers set out to develop a continuous flow platform for the synthesis of cyclobutane 34 (Scheme 11) in order to prepare $>5 \mathrm{~kg}$ /day of the target compound [72]. To achieve this, a multistage approach was executed. This involved focused reaction optimisation, proof of concept through an intermediate scale-up (500 g/day) and finally, the design of a production scale skid ( $>5 \mathrm{~kg} /$ day).

As is often the case when scaling up a reaction to industrial scales, development and optimisation of a product isolation method becomes increasingly important. As previously reported $[70,73]$, the presence of a photosensitiser, benzophenone, is crucial for the reactivity of maleic anhydride with ethylene. Increasing the quantity of benzophenone resulted in increased reaction efficiency, however, a negative impact on crystallisation efficiency (and overall isolated yield) was also observed. Therefore, an amount of $10 \mathrm{~mol} \%$ was chosen to strike the balance between the two parameters. The continuous flow setup consisted of a stream of maleic anhydride/ benzophenone, which was combined with ethylene gas in a mixing tank, with the mixture then passing through a photoreactor (Scheme 12).

While a standard T-piece mixer was sufficient for the intermediate scale $(500 \mathrm{~g} /$ day $)$, as flow rate was increased the presence of ethylene slugs was observed. This slug flow could be eliminated using a mixing tank with a residence time of $30 \mathrm{~min}$. To increase productivity, the internal diameter of the reactor tubing was increased to $10 \mathrm{~mm}$ (compared to the lab scale $\sim 1-2 \mathrm{~mm}$ ). Due to the significantly increased diameter, a 
Scheme 10 Photochemical $[2+$ 2]-cycloaddition of ethylene

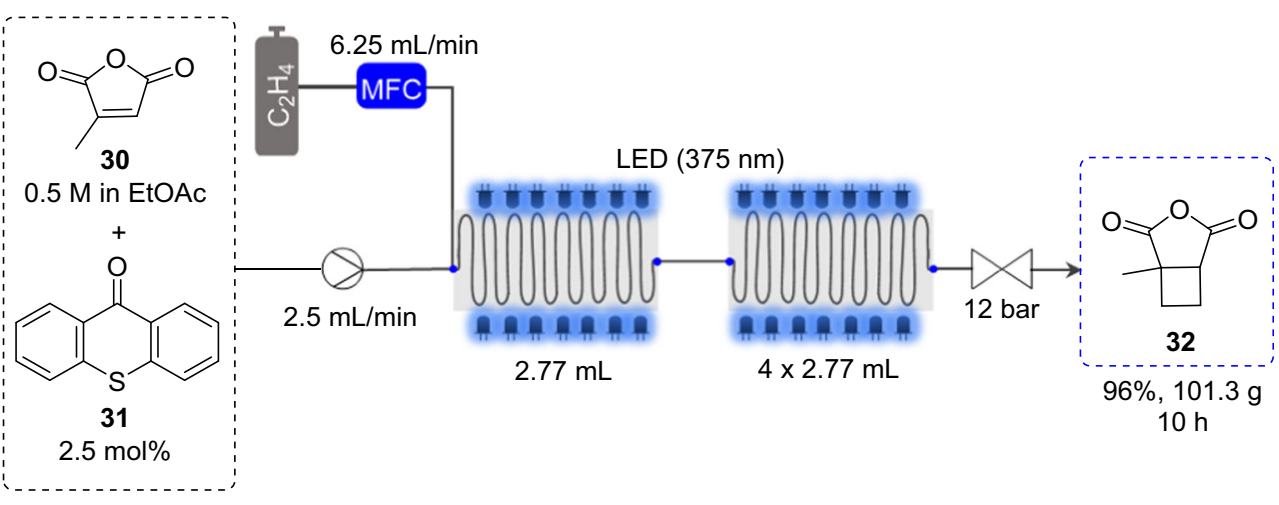

matched increase in lamp power was required. For the intermediate scale setup, $2 \times 300 \mathrm{~W}$ LED panels $(365 \mathrm{~nm})$ were found to be sufficient. However, with the increase in volume to the production scale (from $1.56 \mathrm{~L}$ to $20.76 \mathrm{~L}$ irradiated volume) a corresponding increase in lamp power was required $(6 \times 3 \mathrm{~kW}$ LED panels for production scale). As is the case with any increase in lamp power, significant additional cooling was required to maintain a constant temperature. This was achieved simply by adjusting the flow rate of the cooling medium. The final optimised production scale skid was then run uninterrupted for a period of one week, producing $51.8 \mathrm{~kg}$ ( $7.4 \mathrm{~kg} /$ day) of cyclobutane 34 thus exceeding the initial target of $5 \mathrm{~kg} /$ day with the setup being used to eventually generate $>250 \mathrm{~kg}$ of $\mathbf{3 4}$. While significant safety considerations were required for such a scale, many of the concerns that are associated with such a reaction were minimised using continuous flow chemistry (Scheme 12). This combined with the relatively small footprint (Fig. 9) makes it difficult to develop a safe batch reactor with comparable efficiency.

While highly efficient for carrying out photochemical reactions, the use of FEP reactors can prove problematic on larger scales. While FEP is a highly versatile material, it is not completely UV transparent and any weak spots within

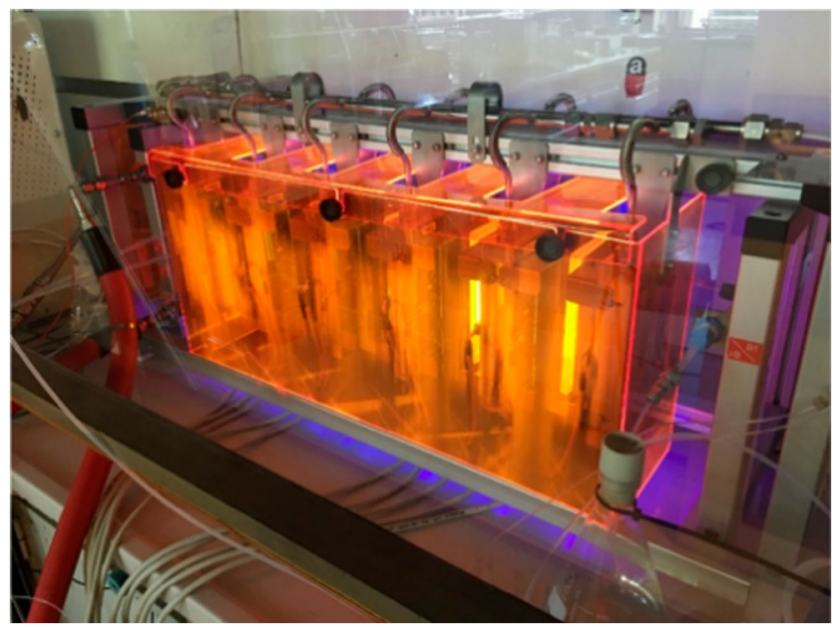

Fig. 8 Corning G1 photoreactor. Reprinted with permission from [70]. Copyright (2019) American Chemical Society the tubing may lead to rupture when operated under pressure. Additionally, reactor fouling can be a common issue [70,73], while oftentimes reversible by flushing solvent through the reactor, this fouling/blocking can be irreversible in some cases. This results in the need to replace the entire length of tubing within the reactor which is highly wasteful on large scale (lengths of tubing exceed hundreds of metres in some cases). In order to mitigate these issues, a collaboration between industrial groups and the Booker-Milburn group developed a quartz reactor which they coined the 'Firefly' reactor (Fig. 10) [20].

The Firefly reactor consists of an array of axially arranged quartz tubes (internal volume $120 \mathrm{~mL}$ ) around a light source. The use of quartz offers the advantage of near-complete UV transparency in addition to increased durability when compared to FEP. A standard high-power medium pressure mercury lamp was used in this case, but the light source could be easily exchanged to suit a range of photochemical reactions. To protect the user from powerful UV radiation, the reactor was placed in a metal jacket, which offered the additional benefit of reflecting any light back towards the reactor. Despite the presence of a cooling jacket surrounding the reactor tubing, the introduction of a metal reflector caused significant overheating. This could be overcome through the introduction of a fan to displace stagnant air caught between the light source and reactor tubing.

In order to test the reactor, a range of [2+2]-cycloaddition reactions were investigated, including the synthesis of "Cookson's dione" (36, Scheme 13) [74]. This involves the intramolecular $[2+2]$-cycloaddition of the ene-dione $\mathbf{3 5}$ which had previously been demonstrated using a FEP reactor [9]. Using the Firefly reactor at $1.5 \mathrm{~kW}$ and a concentration of<smiles>O=C1C=CC(=O)O1</smiles>

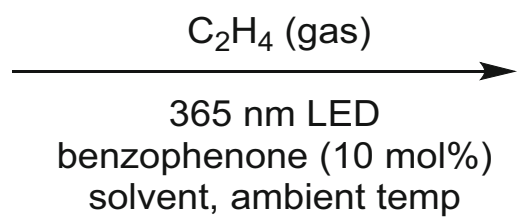<smiles>O=C1OC(=O)[C@H]2CC[C@H]12</smiles>

Scheme 11 Photochemical synthesis of cyclobutane 34 
Scheme 12 Kilogram-scale continuous flow setup. Reprinted with permission from [72]. Copyright (2020) American Chemical Society

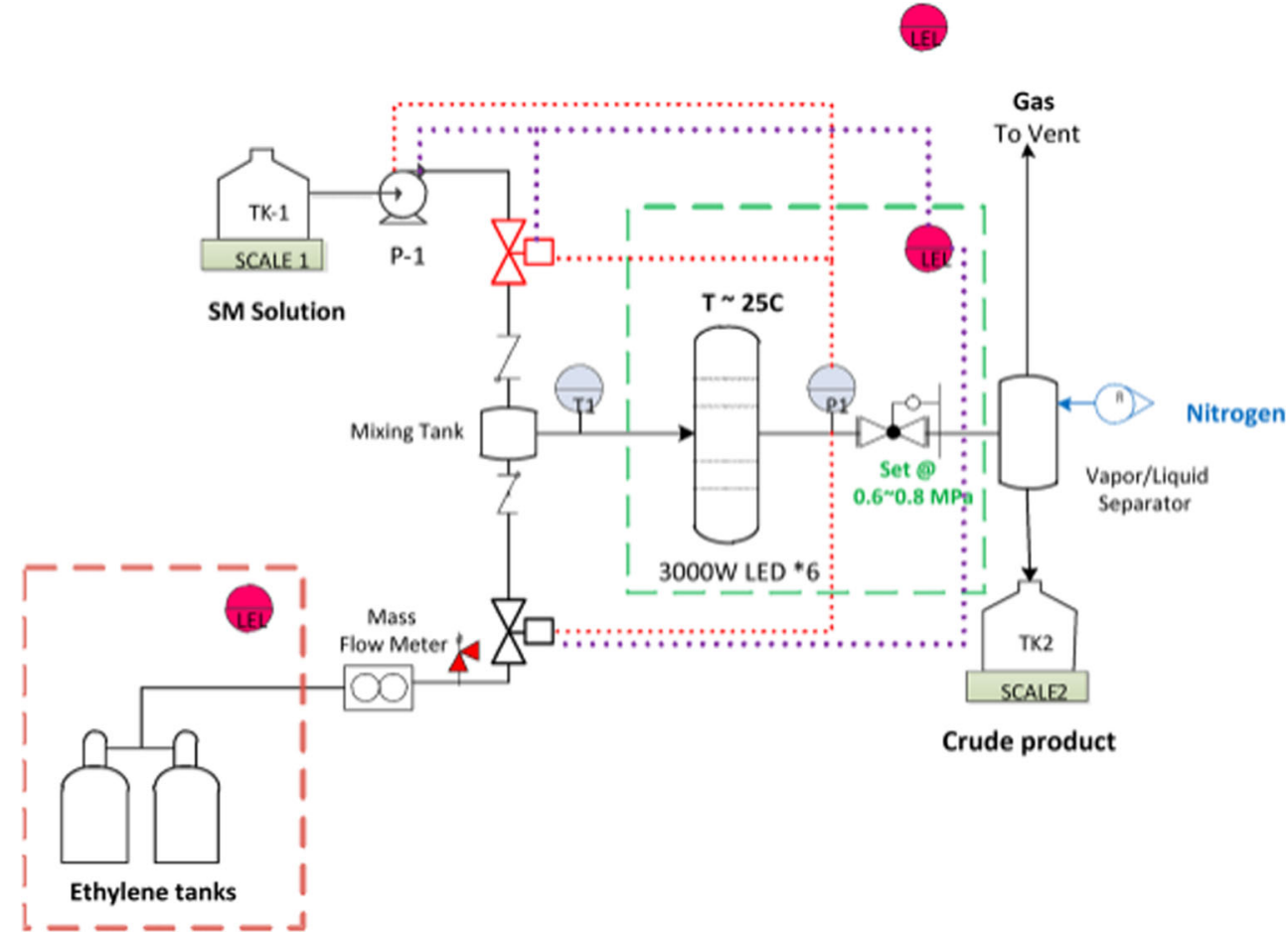

$0.5 \mathrm{M}$ a productivity of $\sim 4 \mathrm{~kg} /$ day could be achieved. Doubling of the concentration and light power corresponded to a doubling of productivity ( $8 \mathrm{~kg} /$ day) which was demonstrated by synthesising $1165 \mathrm{~g}$ of $\mathbf{3 6}$ in just $3.5 \mathrm{~h}$. Based on these results using the full $5 \mathrm{~kW}$ power rating a total productivity of $13 \mathrm{~kg}$ /day could be achieved. Comparison of this methodology to the previously published FEP reactor [9] found the Firefly system to be almost 30\% more power efficient which while not crucial on laboratory scale, is of prime importance for manufacturing.

The majority of novel innovative reactor designs for kilogram-scale continuous flow photochemical synthesis have only been reported over the last decade $[20,61,72$, 75-77]. Many of these involve scaling up reactors that were designed for laboratory scale synthesis, such as the vortex reactor reported by Poliakoff, George and coworkers $[22,76]$. The design of a reactor that utilised a rotating cylinder inside a static cylinder in order to generate Taylor vortices was reported in 2017 [22]. This reactor was used for the photooxidation of citronellol with the required oxygen being supplied by air drawn into the reactor. While innovative, this design was not suitable to larger scales ( $>10 \mathrm{~g} /$ day) due to volume constraints.

In order to develop a system capable of producing kilograms of product, a reactor of approximately 20 times the volume was developed (Fig. 11) [76]. The reactor consisted of a polymer rotor housed within a jacketed filter-tube sealed with a steel base and polymer cap. Inlet pores were bored through the steel base and outlets were found in the polymer cap. Cooling was provided by a recirculating chiller connected to a jacketed glass vessel and a gap of $2 \mathrm{~mm}$ was employed between the glass jacket and polymer rotor giving a total reactor volume of $280 \mathrm{~mL}$. The reactor was irradiated using a light source housed outside the cooling jacket.

To demonstrate the utility of this reactor the photooxidation of citronellol (37) was once again chosen (Scheme 14). In contrast to small scale experiments, air, which provides the required oxygen, could not reliably be drawn in due to the increased volume of the reactor and the reduced rotation speed of the internal rotor.
Fig. 9 Image of photochemical skid. Reprinted with permission from [72]. Copyright (2020) American Chemical Society
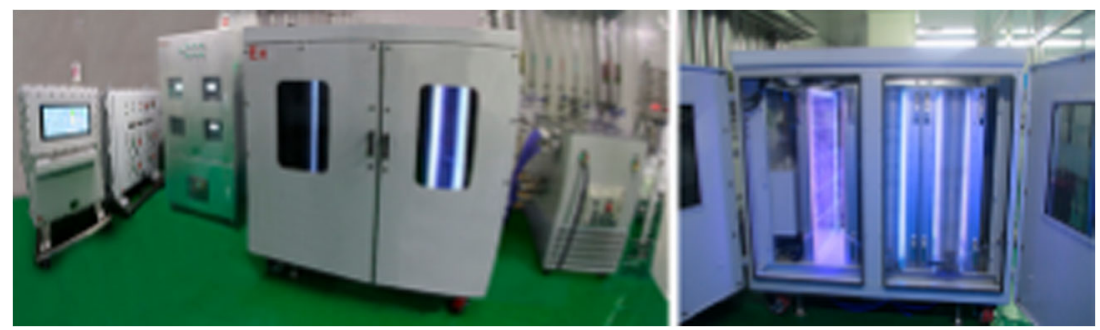
Fig. 10 'Firefly' photochemical reactor (reproduced with permission from [20])
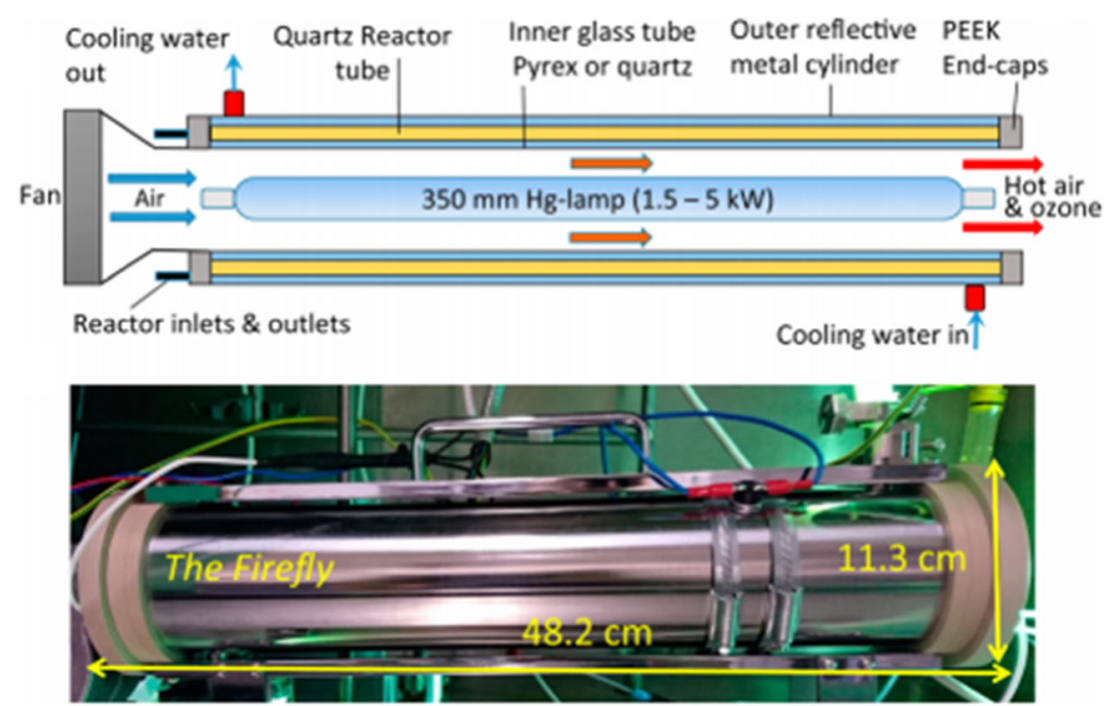

Using a stream of oxygen gas, coupled with a sufficiently high rotation speed, rendered the photoproducts in yields of up to $92 \%$ (both isomers $\mathbf{3 8}$ and $\mathbf{3 9}$ ). In order to determine the rotation speed required for sufficient mixing, various Computational Fluid Dynamics (CFD) calculations were carried out, this aided optimisation of the reaction and is typically an extremely valuable tool in the scaling up of reactions. The optimised conditions used a concentration of $0.2 \mathrm{M}$ with a residence time of $7 \mathrm{~min}$, through which a productivity of $\sim 2 \mathrm{~kg} /$ day could be achieved. This corresponded to a $\sim 10$-fold increase in space-time-yield compared to the previous smallscale reactor. It is worth noting that the $[2+2]$-cycloaddition of "Cookson's dione" (Scheme 13) was also carried out with a productivity comparable to that achieved by the Firefly reactor [20] ( $\sim 7.5 \mathrm{~kg} / \mathrm{day}$ vs $\sim 8 \mathrm{~kg} / \mathrm{day})$, emphasising that similar results can be achieved utilising different reactor designs.

As an alternative approach for carrying out photochemical oxidations in a continuous manner the Noël group recently reported the use of a spinning disk reactor [78]. Biphasic reactions are typically highly mass-transfer dependant, with efficient mixing being required in order to obtain high conversions. In order to achieve efficient mixing in plug flow reactors, supplementary mixing devices are typically required. In order to overcome this requirement, the Noël group opted to use a Rotor-Stator Spinning Disk Reactor (RS-SDR). Using<smiles>O=C1C=CC(=O)C2C3C=CC(C3)C12</smiles>

35

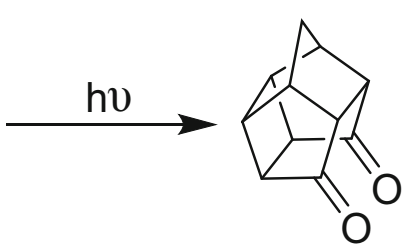

36
Scheme 13 Synthesis of 'Cookson's dione' this class of reactor, extremely efficient mixing can be achieved through modification of the speed at which the internal disk rotates. The reactor consisted of a rotor housed within a $64 \mathrm{~mL}$ reactor, a quartz window was employed to allow the mixture to be irradiated, giving an irradiated volume of $27 \mathrm{~mL} .120 \mathrm{~W}$ white LEDs were utilised to irradiate the reaction mixture.

In order to demonstrate the utility of this class of reactors for photochemical processes, the photo-oxidation of $\alpha$ terpinene was selected (Scheme 9). The setup consisted of a stream of Rose-Bengal and $\alpha$-terpinene, which were combined with a separate stream of $\mathrm{O}_{2}$ gas in a T-piece mixer, before entering the RS-SDR. To monitor the effect of rotation speed on mixing, a high-speed camera was used to image the disk (Fig. 12). Optimisation studies revealed the benefit obtained from high rotation speeds plateaued at approximately $2000 \mathrm{rpm}$, this value decreased with an increase in oxygen concentration, presumably due to the increased excess present in the reaction mixture leading to shortened reaction times. Using the optimised conditions of low concentrations $(0.1 \mathrm{M})$ and high rotation speeds and flow rate $(2000 \mathrm{rpm}$ and $50 \mathrm{~mL} / \mathrm{min}$ ), a productivity approximately 2.6 times higher than the equivalent microflow reactor could be achieved. This equated to a productivity of up to $1.1 \mathrm{~kg} /$ day, with a short residence time of $27 \mathrm{~s}$. In addition to higher productivity, the use of the RS-SDR allows for simple adjustment of mass transfer by modifying the rotational speed of the internal disc. This versatility demonstrates the potential applicability of this system to other mass-transfer limited photochemical processes.

While LED technology has developed significantly over the past decade, there still exists a lack of available highintensity monochromatic LEDs. As an alternative, Harper, Moschetta and co-workers developed a CSTR setup which utilised a $25 \mathrm{~W} 450 \mathrm{~nm}$ fibre coupled laser system [77]. One 
Fig. 11 Kilogram-scale vortex reactor. Reprinted with permission from [76]. Copyright (2020) American Chemical Society
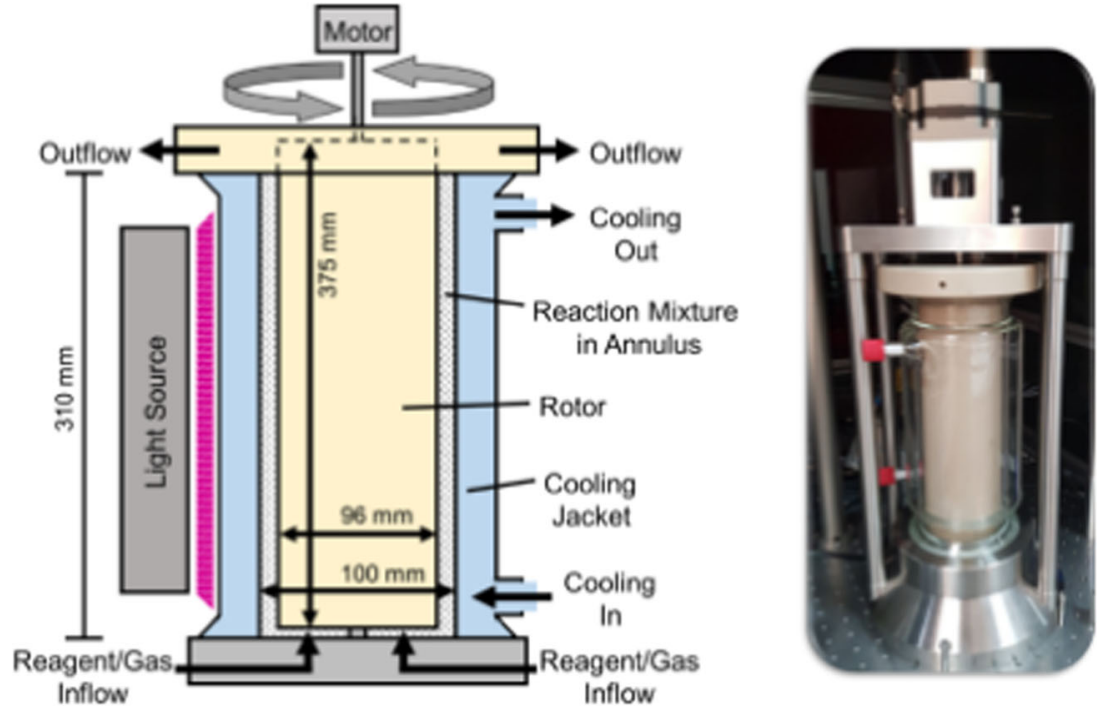

of the disadvantages of tubular flow reactors is the requirement for a fixed fluid volume within a reactor of a fixed length. In contrast, variable fluid volumes can be utilised within a fixed volume CSTR. To overcome this a CSTR setup was designed which could theoretically operate at different volumes. The penetration of light through a reaction mixture is consistently cited as a limitation for scaling up photochemical reactions in batch. However, this limitation can also apply to CSTRs with the Beer-Lambert law describing the relationship between light absorption and path length (reactor depth).

In order to explore this correlation and demonstrate the use of high-power lasers as an alternative light source, the iridiumnickel co-catalysed C-N coupling of aryl bromides [79] was investigated (Scheme 15). Experiments in batch found that the absorption of light by the reaction mixture was highly dependent on the catalyst concentrations, with over $99 \%$ of the incident light being absorbed at $1 \mathrm{~cm}$ of depth with a catalyst concentration of $6 \mathrm{mM}$. Through reaction optimisation, the optimal catalyst concentration was found to be $2 \mathrm{mM}$, at which the light could penetrate a depth of $5 \mathrm{~cm}$. This depth was the basis for the subsequent CSTR parameters. The final CSTR consisted of inlet and outlet pumps, a $25 \mathrm{~W}$ fibre optic laser and a beam expander. The reactor volume was kept constant at $100 \mathrm{~mL}$ with a depth of $5 \mathrm{~cm}$ (Scheme 15). Using this setup, the reactor was run at steady state for $32 \mathrm{~h}$ yielding $1.54 \mathrm{~kg}$ ( $85 \%$ yield) of $\mathbf{4 2}$. This corresponded to a productivity of $1.2 \mathrm{~kg} /$ day, which could be increased further using more powerful lasers or a stream of cascading CSTRs. After this long run, a fine coating was observed on the reactor walls which was not found to measurably affect the reactor performance. This is to be expected as the fouling did not affect light absorption, however, it is worth noting that reactor fouling using a typical tubular flow reactor typically results in diminished yields. In addition to demonstrating the applicability of alternative light sources, the use of CSTRs could also allow for photochemical transformations, which involve solids, on kilogram scale.

Another approach to the scale up of the same reaction, using high-power LEDs was investigated by Lévesque, Di Maso and co-workers [80]. Having previously designed a large volume photoreactor capable of throughputs up to $10 \mathrm{~kg} /$ day [81], a smaller footprint reactor which maintained similar throughput was desired. This smaller reactor was built to fit within a 5.5-gal ( $\sim 20 \mathrm{~L})$ aquarium and consisted of FEP tubing (total volume $890 \mathrm{~mL}$ ) submerged in a recirculating water bath for temperature control. Panels consisting of fifteen

Scheme 14 Photo-oxidation of citronellol<smiles>CC(C)=CCCC(C)CCO</smiles>

37

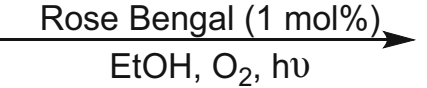<smiles>CC(C=CCC(C)OO)CCO</smiles><smiles>C=C(C)C(CCC(C)CCO)OO</smiles> 


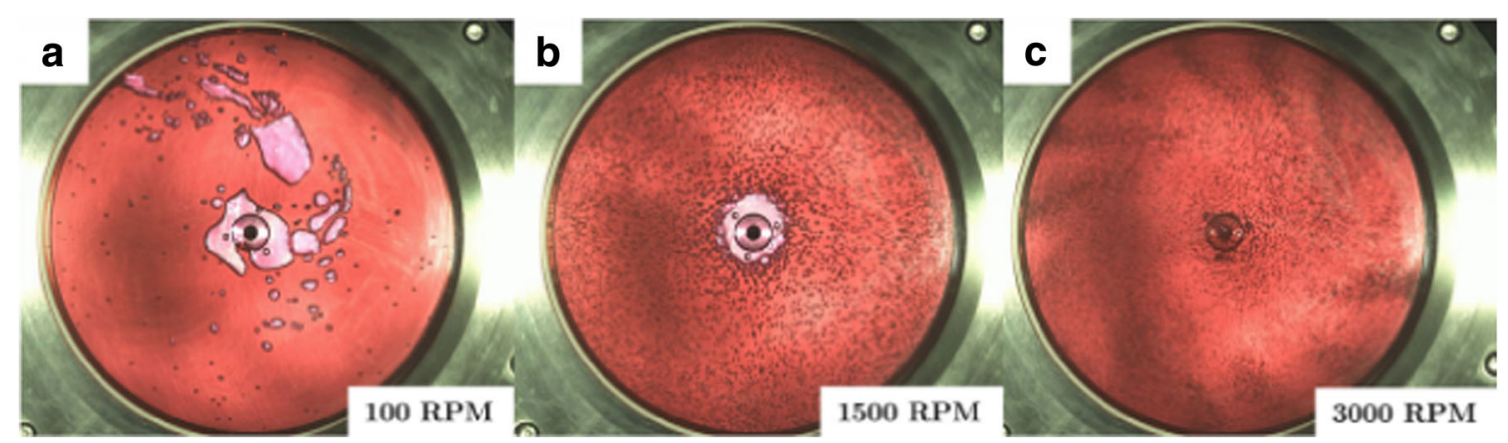

Fig. 12 Comparison of rotation speeds in RS-SDR (reproduced with permission from [78])

$100 \mathrm{~W}$ LED chips (440-450 nm) were placed either side of the reactor, with cooling being achieved by flowing water through copper piping embedded in the panels (Scheme 16). To efficiently scale up a reaction, light absorption by the reaction mixture must be maximised. This was done by increasing the tubing diameter from 3.18 to $7.94 \mathrm{~mm}$ which significantly increased productivity while maintaining complete light transmittance through the solution. Using the optimised conditions (concentration $0.4 \mathrm{M}$ ), the reaction was run for $130 \mathrm{~min}$ at steady state, producing $1.14 \mathrm{~kg}$ of product $(90 \%$ assay yield). This corresponds to a productivity of $12.6 \mathrm{~kg} /$ day, higher than the previous larger footprint reactor [81]. Additionally, the authors noted that this throughput could be increased significantly, at the cost of conversion, by increasing the flow rate from 105 to $925 \mathrm{~mL} / \mathrm{min}$. Using these conditions $1.12 \mathrm{~kg}$ ( $42 \%$ assay yield) of product was obtained in just $37 \mathrm{~min}$ (43.4 kg/day).

Bromination reactions represent an important transformation in the synthesis of building blocks for the pharmaceutical and fine chemical industries [82]. A range of methodologies exist, including various photochemical transformations using either molecular bromine or an alternative bromine source [83]. The direct use of molecular bromine for these reactions is unfavourable due to safety concerns on larger scales, and while $N$-bromosuccinimide can act as a safer alternative [84], its use is not ideal due to poorer reactivity and modest atom economy. Another strategy involves the in-situ generation of bromine [85], which was recently explored by the Kappe group [75]. The relatively green reaction between $\mathrm{NaBrO}_{3}$ and hydrobromic acid provides access to $\mathrm{Br}_{2}$ with the formation of water as a by-product [86]. However, due to the exothermic nature of the reaction, precise temperature control is required.

Initial reports of the process intensified photochemical benzyl bromination, using in-situ generated bromine, within a lab scale Corning photochemical reactor (volume: $2.8 \mathrm{~mL}$ ) provided a throughput of $300 \mathrm{~g} / \mathrm{h}$ [86]. To further scale up to pilot-scale the significantly larger Corning G3 reactor (volume: $50 \mathrm{~mL}$ ) was employed. The setup consisted of two fluidic modules (FMs); one to carry out the photochemical reaction and a subsequent FM for quenching of excess $\mathrm{Br}_{2}$ with sodium thiosulfate. Separate streams of neat 2,6dichlorotoluene substrate (43) and aqueous $\mathrm{NaBrO}_{3}$ were combined prior to being mixed with hydrobromic acid, to form $\mathrm{Br}_{2}$ which subsequently passed through the photochemical reactor (Scheme 17). LED panels (405 nm) were used to irradiate the mixture with a lamp temperature of $<20^{\circ} \mathrm{C}$ being maintained using a Lauda Proline RP 890 thermostat, while a separate thermostat was used to regulate reactor temperature. Gear pumps were used to dose the non-acidic streams while a metal-free FUJI pump was utilised for administration of $\mathrm{HBr}$. A small webcam was also positioned within the reactor box
Scheme 15 Laser-mediated photochemical aryl amination

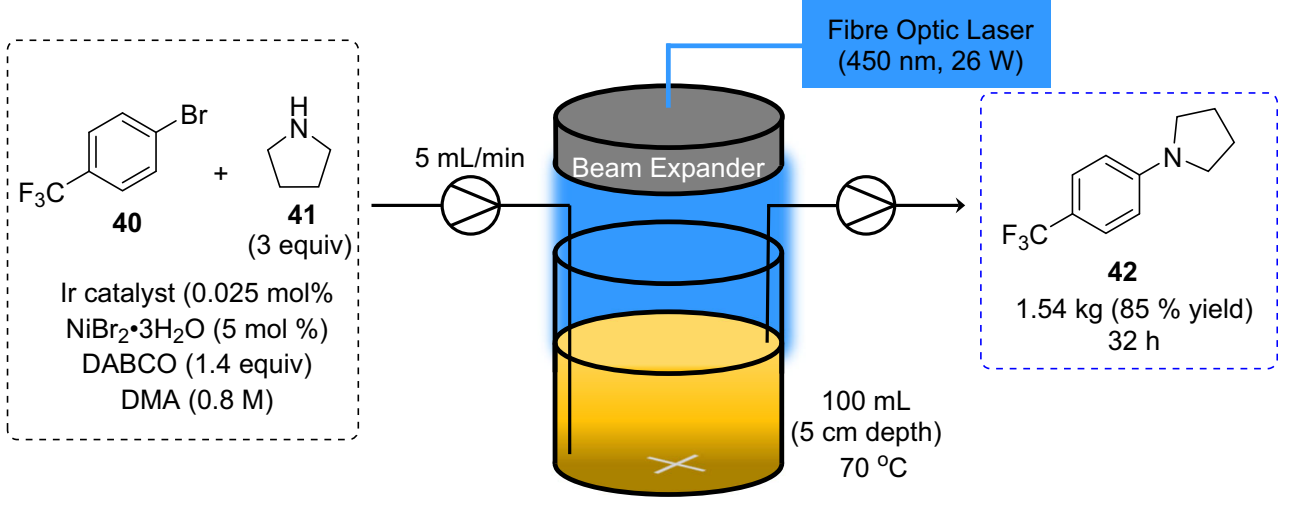


Scheme 16 LED-mediated photochemical aryl amination

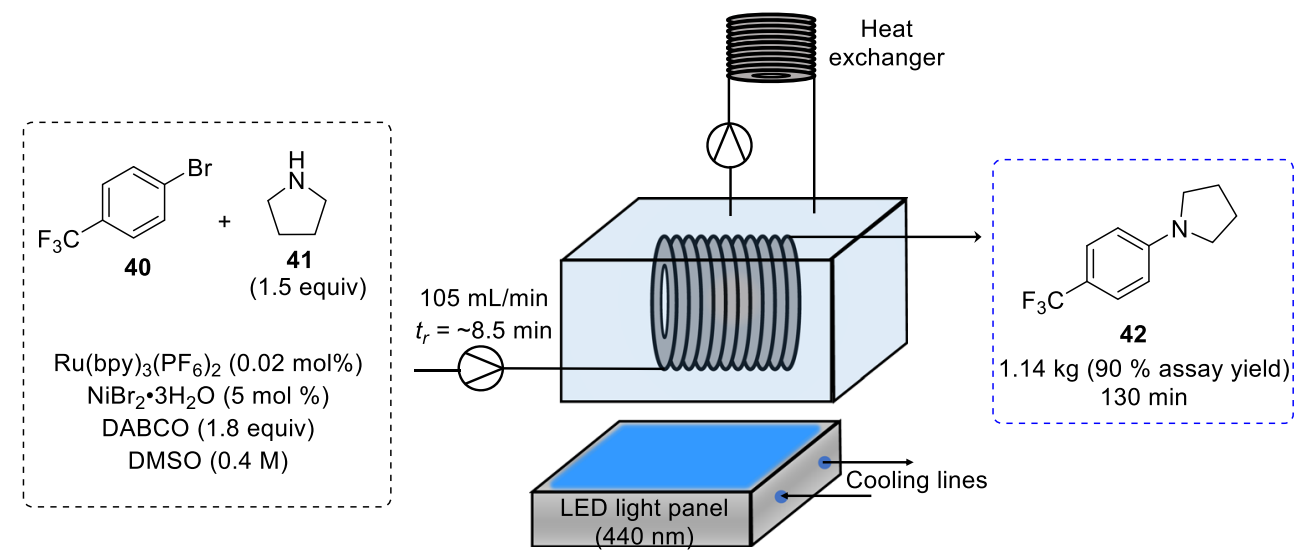

\section{Conclusions and future directions}

While this review is not intended to provide a comprehensive list of examples, it is clear that through the advancement of available technology the scalability of photochemical reactions has changed over the past decade. Prior to the last five years there were scarce reports of kilogram scale photochemical processes carried out in continuous flow, however, this has been rectified in recent years, largely driven by the development of innovative photochemical reactors. While flow photochemistry may present various advantages over its batch counterpart, both methodologies can be used in a synergistic manner. Batch offers the advantage of simple real time analysis through common laboratory methods such as TLC and HPLC, providing powerful insight to a process. This allows for relatively rapid screening of conditions on a small scale, which may not be possible in flow due to the prohibitive cost of analytical equipment required to perform similar monitoring of reactions in continuous flow. Additionally, various photochemical processes can be scaled up to the decagram scale in batch with ease. However, this is generally limited to reactions that do not suffer from photochemical degradation and other factors that can be mitigated though flow processing, thus reducing the complexity of chemical structures accessible through such batch-based methodology. tinuous processing when compared to batch, in particular for processes that require both high mass transfer and photon flux.

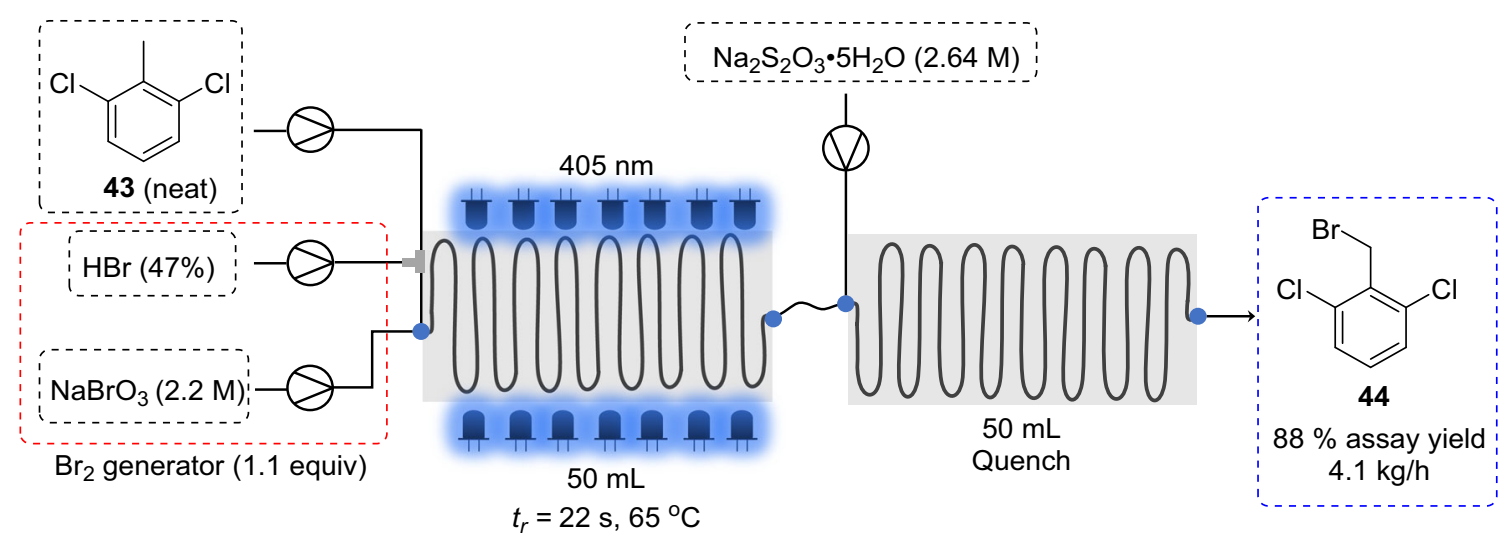

Scheme 17 Kilogram-scale benzylic bromination 
The statement that batch and flow photochemistry exhibit similar performance when corrected for factors such as light power-to-surface area, as concluded by Booker-Milburn and co-workers in 2014 [9], is still relevant. However, this may be limited to uncatalysed cyclisation reactions. In contrast, several of the discussed reactions, with higher comparable complexity, seem to benefit significantly from continuous flow. Additionally, it is evident that practical scalability is achieved more easily through the use of continuous flow technology. Various examples have been presented on a kilogram scale using small-footprint reactors, where the equivalent batch process would require prohibitively large vessels and light sources. The increase in availability of high powered nearmonochromatic light sources in addition to the advancements in continuous flow reactor design has led to an increased adoption of photochemistry on larger scales.

Previously, such scales may have been limited to relatively simple reactions, such as photocyclisations or oxygenations, however, recent reports have expanded to more complex processes such as photoredox catalysis. This expansion in reaction scope is only expected to continue due to the availability of different reactor designs as discussed throughout this review. While some requirement for reaction reoptimisation typically remains, the use of either larger reactors or several modular reactors in parallel or series presents an attractive strategy to mitigate issues arising from differences in fluid mechanics for larger reactors.

Many of the processes discussed have been scaled up progressively over a period of time, with their intial smaller scale reports emerging in the preceeding years. It is worth noting that reactions carried out on kilogram scale are typically reserved for products which are deemed commercially important and therefore required in large amounts. As a result, few reports on such a large scale exist. However, this does not mitigate the scalability of novel photochemical processes, as similar results are expected to be achievable through careful reactor design. One can expect the expansion of accessible chemical space driven by new photochemical reactions to continue, and their subsequent scale up to be accelerated through the use of the current range of reactor designs. The ever evolving landscape of continuous flow photochemical reactors presents many exciting oppurtunities which we hope to see materialise over the coming years.

Acknowledgements The School of Chemistry at UCD is gratefully acknowledged for providing a Research Demonstratorship (to KD) and support of our research programme.

Funding Open Access funding provided by the IReL Consortium.

\section{Declarations}

Conflict of interest We declare that no conflict of interest exists.
Open Access This article is licensed under a Creative Commons Attribution 4.0 International License, which permits use, sharing, adaptation, distribution and reproduction in any medium or format, as long as you give appropriate credit to the original author(s) and the source, provide a link to the Creative Commons licence, and indicate if changes were made. The images or other third party material in this article are included in the article's Creative Commons licence, unless indicated otherwise in a credit line to the material. If material is not included in the article's Creative Commons licence and your intended use is not permitted by statutory regulation or exceeds the permitted use, you will need to obtain permission directly from the copyright holder. To view a copy of this licence, visit http://creativecommons.org/licenses/by/4.0/.

\section{References}

1. Sambiagio C, Noël T (2020) Flow photochemistry: Shine some light on those tubes! Trends Chem 2:92-106. https://doi.org/10. 1016/j.trechm.2019.09.003

2. Rehm TH (2020) Flow photochemistry as a tool in organic synthesis. Chem Eur J 26:16952-16974. https://doi.org/10.1002/chem. 202000381

3. Di Filippo M, Bracken C, Baumann M (2020) Continuous flow photochemistry for the preparation of bioactive molecules. Molecules 25:356. https://doi.org/10.3390/molecules/25020356

4. J-i Y, Kim H, Nagaki A (2017) "Impossible" chemistries based on flow and micro. J Flow Chem JFChem 7:60-64. https://doi.org/10. 1556/1846.2017.00017

5. Shaw MH, Twilton J, MacMillan DWC (2016) Photoredox catalysis in organic chemistry. J Org Chem 81:6898-6926. https://doi. org/10.1021/acs.joc.6b01449

6. McAtee RC, McClain EJ, Stephenson CRJ (2019) Illuminating Photoredox catalysis. Trends Chem 1:111-125. https://doi.org/10. 1016/j.trechm.2019.01.008

7. König B (2017) Photocatalysis in organic synthesis - past, present, and future. Eur J Org Chem 2017:1979-1981. https://doi.org/10. 1002/ejoc.201700420

8. Kayahan E, Jacobs M, Braeken L, Thomassen LCJ, Kuhn S, van Gerven T, Leblebici ME (2020) Dawn of a new era in industrial photochemistry: the scale-up of micro- and mesostructured photoreactors. Beilstein J Org Chem 16:2484-2504. https://doi. org/10.3762/bjoc. 16.202

9. Elliott LD, Knowles JP, Koovits PJ, Maskill KG, Ralph MJ, Lejeune G, Edwards LJ, Robinson RI, Clemens IR, Cox B, Pascoe DD, Koch G, Eberle M, Berry MB, Booker-Milburn KI (2014) Batch versus flow photochemistry: a revealing comparison of yield and productivity. Chem Eur J 20:15226-15232. https://doi. org/10.1002/chem.201404347

10. Abdiaj I, Alcázar J (2017) Improving the throughput of batch photochemical reactions using flow: dual photoredox and nickel catalysis in flow for $\mathrm{C}(\mathrm{sp} 2) \mathrm{C}(\mathrm{sp} 3)$ cross-coupling. Bioorg Med Chem 25:6190-6196. https://doi.org/10.1016/j.bmc.2016.12.041

11. Bracken C, Baumann M (2020) Development of a continuous flow Photoisomerization reaction converting Isoxazoles into diverse Oxazole products. J Org Chem 85:2607-2617. https://doi.org/10. 1021/acs.joc.9b03399

12. Chen Y, Cantillo D, Kappe CO (2019) Visible light-promoted Beckmann rearrangements: separating sequential photochemical and thermal phenomena in a continuous flow reactor. Eur J Org Chem 2019:2163-2171. https://doi.org/10.1002/ejoc.201900231

13. Laudadio G, Deng Y, van der Wal K, Ravelli D, Nuño M, Fagnoni M, Guthrie D, Sun Y, Noël T (2020) C(sp3)-H functionalizations 
of light hydrocarbons using decatungstate photocatalysis in flow. Science 369:92-96. https://doi.org/10.1126/science.abb4688

14. Di Filippo M, Baumann M (2020) Continuous flow synthesis of Quinolines via a scalable tandem Photoisomerization-cyclization process. Eur J Org Chem 2020:6199-6211. https://doi.org/10. 1002/ejoc.202000957

15. Bianchi P, Petit G, Monbaliu J-CM (2020) Scalable and robust photochemical flow process towards small spherical gold nanoparticles. React Chem Eng 5:1224-1236. https://doi.org/10.1039/ D0RE00092B

16. Lebl R, Cantillo D, Kappe CO (2019) Continuous generation, inline quantification and utilization of nitrosyl chloride in photonitrosation reactions. React Chem Eng 4:738-746. https:// doi.org/10.1039/C8RE00323H

17. Chen Y, de Frutos O, Mateos C, Rincon JA, Cantillo D, Kappe CO (2018) Continuous flow photochemical Benzylic Bromination of a key intermediate in the synthesis of a 2-Oxazolidinone. ChemPhotoChem 2:906-912. https://doi.org/10.1002/cptc. 201800114

18. Chen Y, May O, Blakemore DC, Ley SV (2019) A Photoredox coupling reaction of Benzylboronic esters and carbonyl compounds in batch and flow. Org Lett 21:6140-6144. https://doi.org/10.1021/ acs.orglett.9b02307

19. Lima F, Grunenberg L, Rahman HBA, Labes R, Sedelmeier J, Ley SV (2018) Organic photocatalysis for the radical couplings of boronic acid derivatives in batch and flow. Chem Commun 54:56065609. https://doi.org/10.1039/C8CC02169D

20. Elliott LD, Berry M, Harji B, Klauber D, Leonard J, BookerMilburn KI (2016) A small-footprint, high-capacity flow reactor for UV photochemical synthesis on the kilogram scale. Org Process Res Dev 20:1806-1811. https://doi.org/10.1021/acs.oprd. $6 \mathrm{~b} 00277$

21. Clark CA, Lee DS, Pickering SJ, Poliakoff M, George MW (2018) UV PhotoVap: demonstrating how a simple and versatile reactor based on a conventional rotary evaporator can be used for UV photochemistry. Org Process Res Dev 22:595-599. https://doi. org/10.1021/acs.oprd.8b00037

22. Lee DS, Amara Z, Clark CA, Xu Z, Kakimpa B, Morvan HP, Pickering SJ, Poliakoff M, George MW (2017) Continuous photo-oxidation in a vortex reactor: efficient operations using air drawn from the laboratory. Org Process Res Dev 21:1042-1050. https://doi.org/10.1021/acs.oprd.7b00153

23. Britton J, Stubbs KA, Weiss GA, Raston CL (2017) Vortex fluidic chemical transformations. Chem Eur J 23:13270-13278. https:// doi.org/10.1002/chem. 201700888

24. Debrouwer W, Kimpe W, Dangreau R, Huvaere K, Gemoets HPL, Mottaghi M, Kuhn S, Van Aken K (2020) Ir/Ni Photoredox dual catalysis with Heterogeneous Base enabled by an oscillatory plug flow Photoreactor. Org Process Res Dev 24:2319-2325. https://doi. org/10.1021/acs.oprd.0c00150

25. Rosso C, Gisbertz S, Williams JD, Gemoets HPL, Debrouwer W, Pieber B, Kappe CO (2020) An oscillatory plug flow photoreactor facilitates semi-heterogeneous dual nickel/carbon nitride photocatalytic C-N couplings. React Chem Eng 5:597-604. https://doi.org/ 10.1039/D0RE00036A

26. Reckenthäler M, Griesbeck AG (2013) Photoredox catalysis for organic syntheses. Adv Synth Catal 355:2727-2744. https://doi. org/10.1002/adsc.201300751

27. Xuan J, Xiao W-J (2012) Visible-light Photoredox catalysis. Angew Chem Int Ed 51:6828-6838. https://doi.org/10.1002/anie. 201200223

28. Romero NA, Nicewicz DA (2016) Organic Photoredox catalysis. Chem Rev 116:10075-10166. https://doi.org/10.1021/acs. chemrev.6b00057

29. Zhang $\mathrm{H}$, Lei A (2018) Visible-light-induced $\mathrm{C}-\mathrm{H}$ functionalization and $\mathrm{C}-\mathrm{C} / \mathrm{C}-\mathrm{X}$ bond-forming oxidative cross- coupling reactions. Asian J Org Chem 7:1164-1177. https://doi. org/10.1002/ajoc.201800214

30. Petzold D, Giedyk M, Chatterjee A, König B (2020) A retrosynthetic approach for Photocatalysis. Eur J Org Chem 2020:1193-1244. https://doi.org/10.1002/ejoc.201901421

31. Ravelli D, Protti S, Fagnoni M (2016) Carbon-carbon bond forming reactions via Photogenerated intermediates. Chem Rev 116:9850-9913. https://doi.org/10.1021/acs.chemrev.5b00662

32. Zhang P, Le CC, MacMillan DWC (2016) Silyl radical activation of alkyl halides in Metallaphotoredox catalysis: a unique pathway for cross-electrophile coupling. J Am Chem Soc 138:8084-8087. https://doi.org/10.1021/jacs.6b04818

33. Pomberger A, Mo Y, Nandiwale KY, Schultz VL, Duvadie R, Robinson RI, Altinoglu EI, Jensen KF (2019) A continuous stirred-tank reactor (CSTR) Cascade for handling solid-containing photochemical reactions. Org Process Res Dev 23:2699-2706. https://doi.org/10.1021/acs.oprd.9b00378

34. Mo Y, Jensen KF (2016) A miniature CSTR cascade for continuous flow of reactions containing solids. React Chem Eng 1:501-507. https://doi.org/10.1039/C6RE00132G

35. Santandrea J, Minozzi C, Cruché C, Collins SK (2017) Photochemical dual-catalytic synthesis of Alkynyl sulfides. Angew Chem Int Ed 56:12255-12259. https://doi.org/10.1002/ anie. 201705903

36. Luo J, Zhang J (2016) Donor-acceptor Fluorophores for visiblelight-promoted organic synthesis: Photoredox/Ni dual catalytic C(sp3)-C(sp2) cross-coupling. ACS Catal 6:873-877. https://doi. org/10.1021/acscatal.5b02204

37. Corey EJ, Streith J (1964) Internal Photoaddtion reactions of 2Pyrone and N-Methyl-2-pyridone: a new synthetic approach to Cyclobutadiene. J Am Chem Soc 86:950-951. https://doi.org/10. 1021/ja01059a059

38. Luparia M, Oliveira MT, Audisio D, Frébault F, Goddard R, Maulide N (2011) Catalytic asymmetric Diastereodivergent Deracemization. Angew Chem Int Ed 50:12631-12635. https:// doi.org/10.1002/anie.201106321

39. Souris C, Frébault F, Patel A, Audisio D, Houk KN, Maulide N (2013) Stereoselective synthesis of Dienyl-carboxylate building blocks: formal synthesis of Inthomycin C. Org Lett 15:32423245. https://doi.org/10.1021/ol401226y

40. Souris C, Luparia M, Frébault F, Audisio D, Farès C, Goddard R, Maulide N (2013) An atom-economical and Stereoselective domino synthesis of functionalised Dienes. Chem Eur J 19:6566-6570. https://doi.org/10.1002/chem.201300776

41. Frébault F, Luparia M, Oliveira MT, Goddard R, Maulide N (2010) A versatile and Stereoselective synthesis of functionalized Cyclobutenes. Angew Chem Int Ed 49:5672-5676. https://doi. org/10.1002/anie.201000911

42. Williams JD, Otake Y, Coussanes G, Saridakis I, Maulide N, Kappe CO (2019) Towards a scalable synthesis of 2-Oxabicyclo[2.2.0]hex5-en-3-one using flow photochemistry. ChemPhotoChem 3:229 232. https://doi.org/10.1002/cptc.201900017

43. Kärkäs MD, Porco JA, Stephenson CRJ (2016) Photochemical approaches to complex Chemotypes: applications in natural product synthesis. Chem Rev 116:9683-9747. https://doi.org/10.1021/acs. chemrev. 5 b00760

44. Bach T, Hehn JP (2011) Photochemical reactions as key steps in natural product synthesis. Angew Chem Int Ed 50:1000-1045. https://doi.org/10.1002/anie.201002845

45. Yueh H, Gao Q, Porco JA, Beeler AB (2017) A photochemical flow reactor for large scale syntheses of aglain and rocaglate natural product analogues. Bioorg Med Chem 25:6197-6202. https://doi. org/10.1016/j.bmc.2017.06.010

46. Gerard B, Jones Ii G, Porco Jr JA (2004) A biomimetic approach to the rocaglamides employing photogeneration of oxidopyryliums 
derived from 3-hydroxyflavones. J Am Chem Soc 126:13620 13621. https://doi.org/10.1021/ja044798o

47. Gerard B, Cencic R, Pelletier J, Porco Jr JA (2007) Enantioselective synthesis of the complex Rocaglate (-)-Silvestrol. Angew Chem Int Ed 46:7831-7834. https://doi.org/10.1002/anie.200702707

48. Lovering F (2013) Escape from flatland 2: complexity and promiscuity. MedChemComm 4:515-519. https://doi.org/10.1039/ C2MD20347B

49. Lovering F, Bikker J, Humblet C (2009) Escape from flatland: increasing saturation as an approach to improving clinical success. J Med Chem 52:6752-6756. https://doi.org/10.1021/jm901241e

50. Cox B, Booker-Milburn KI, Elliott LD, Robertson-Ralph M, Zdorichenko V (2019) Escaping from flatland: [2 + 2] Photocycloaddition; Conformationally constrained sp3-rich scaffolds for Lead generation. ACS Med Chem Lett 10:1512-1517. https://doi.org/10.1021/acsmedchemlett.9b00409

51. Blanco-Ania D, Gawade SA, Zwinkels LJL, Maartense L, Bolster MG, Benningshof JCJ, Rutjes FPJT (2016) Rapid and scalable access into strained scaffolds through continuous flow photochemistry. Org Process Res Dev 20:409-413. https://doi.org/10.1021/ acs.oprd.5b00354

52. Elliott LD, Knowles JP, Stacey CS, Klauber DJ, Booker-Milburn KI (2018) Using batch reactor results to calculate optimal flow rates for the scale-up of UV photochemical reactions. React Chem Eng 3: 86-93. https://doi.org/10.1039/C7RE00193B

53. Kanaoka Y, Hatanaka Y (1976) Photoinduced reactions. XXIV Photochemistry of the imide system I Synthetic photochemistry with the imide system Norrish type II cyclization of alicyclic imides. J Org Chem 41:400-401. https://doi.org/10.1021/ jo00864a058

54. Mooney B, Prager R, Ward A (1981) Central nervous system active compounds. X Some aspects of the photochemical rearrangement of N-alkylsuccinimides to hexahydroazepinones. Aust J Chem 34: 2695-2700. https://doi.org/10.1071/CH9812695

55. Nair V, Vellalath S, Babu BP (2008) Recent advances in carboncarbon bond-forming reactions involving homoenolates generated by NHC catalysis. Chem Soc Rev 37:2691-2698. https://doi.org/ 10.1039/B719083M

56. Li C-J (2009) Cross-Dehydrogenative coupling (CDC): exploring $\mathrm{C}-\mathrm{C}$ bond formations beyond functional group transformations. Acc Chem Res 42:335-344. https://doi.org/10.1021/ar800164n

57. Abdiaj I, Fontana A, Gomez MV, de la Hoz A, Alcázar J (2018) Visible-light-induced nickel-catalyzed Negishi cross-couplings by exogenous-photosensitizer-free Photocatalysis. Angew Chem Int Ed 57:8473-8477. https://doi.org/10.1002/anie.201802656

58. Abdiaj I, Horn CR, Alcazar J (2019) Scalability of visible-lightinduced nickel Negishi reactions: a combination of flow photochemistry, use of solid reagents, and in-line NMR monitoring. J Org Chem 84:4748-4753. https://doi.org/10.1021/acs.joc.8b02358

59. Gillis EP, Eastman KJ, Hill MD, Donnelly DJ, Meanwell NA (2015) Applications of fluorine in medicinal chemistry. J Med Chem 58:8315-8359. https://doi.org/10.1021/acs.jmedchem. $5 \mathrm{~b} 00258$

60. Müller K, Faeh C, Diederich F (2007) Fluorine in pharmaceuticals: looking beyond intuition. Science 317:1881. https://doi.org/10. 1126/science. 1131943

61. Beatty Joel W, Douglas James J, Miller R, McAtee Rory C, Cole Kevin P, Stephenson Corey RJ (2016) Photochemical Perfluoroalkylation with pyridine $\mathrm{N}$-oxides: mechanistic insights and performance on a kilogram scale. Chem 1:456-472. https:// doi.org/10.1016/j.chempr.2016.08.002
62. Beatty JW, Douglas JJ, Cole KP, Stephenson CRJ (2015) A scalable and operationally simple radical trifluoromethylation. Nat Commun 6. https://doi.org/10.1038/ncomms8919

63. Hook BDA, Dohle W, Hirst PR, Pickworth M, Berry MB, BookerMilburn KI (2005) A practical flow reactor for continuous organic photochemistry. J Org Chem 70:7558-7564. https://doi.org/10. 1021/jo050705p

64. Cambié D, Bottecchia C, Straathof NJW, Hessel V, Noël T (2016) Applications of continuous-flow photochemistry in organic synthesis, material science, and water treatment. Chem Rev 116:1027610341. https://doi.org/10.1021/acs.chemrev.5b00707

65. Rehm TH (2020) Reactor technology concepts for flow photochemistry. ChemPhotoChem 4:235-254. https://doi.org/10.1002/ cptc. 201900247

66. Clark CA, Lee DS, Pickering SJ, Poliakoff M, George MW (2016) A simple and versatile reactor for photochemistry. Org Process Res Dev 20:1792-1798. https://doi.org/10.1021/acs.oprd.6b00257

67. Günther SO, Ziegler K (1944) Die Synthese des Ascaridols. Naturwissenschaften 32:157-157. https://doi.org/10.1007/ BF01467891

68. Lamberts JJM, Neckers DC (1985) Rose Bengal derivatives as singlet oxygen sensitizers. Tetrahedron 41:2183-2190. https://doi. org/10.1016/S0040-4020(01)96591-3

69. Poplata S, Tröster A, Zou Y-Q, Bach T (2016) Recent advances in the synthesis of Cyclobutanes by olefin [2+2] Photocycloaddition reactions. Chem Rev 116:9748-9815. https://doi.org/10.1021/acs. chemrev.5b00723

70. Williams JD, Nakano M, Gérardy R, Rincón JA, de Frutos Ó, Mateos C, Monbaliu J-CM, Kappe CO (2019) Finding the perfect match: a combined computational and experimental study toward efficient and scalable photosensitized [2 +2] Cycloadditions in flow. Org Process Res Dev 23:78-87. https://doi.org/10.1021/acs. oprd. 8 b00375

71. Mallia CJ, Baxendale IR (2016) The use of gases in flow synthesis. Org Process Res Dev 20:327-360. https://doi.org/10.1021/acs. oprd.5b00222

72. Beaver MG, E-x Z, Z-q L, Zheng S-y, Wang B, Lu J-p, Tao J, Gonzalez M, Jones S, Tedrow JS (2020) Development and execution of a production-scale continuous [2 +2$]$ Photocycloaddition. Org Process Res Dev 24:2139-2146. https://doi.org/10.1021/acs. oprd.0c00185

73. Corcoran EB, Lévesque F, McMullen JP, Naber JR (2018) Studies toward the scaling of gas-liquid Photocycloadditions. ChemPhotoChem 2:931-937. https://doi.org/10.1002/cptc. 201800098

74. Cookson RC, Crundwell E, Hill RR, Hudec J (1964) 586. Photochemical cyclisation of diels-alder adducts. J Chem Soc (Resumed):3062-3075. https://doi.org/10.1039/JR9640003062

75. Steiner A, Roth PMC, Strauss FJ, Gauron G, Tekautz G, Winter M, Williams JD, Kappe CO (2020) Multikilogram per hour continuous photochemical Benzylic Brominations applying a smart dimensioning scale-up strategy. Org Process Res Dev 24:2208-2216. https:// doi.org/10.1021/acs.oprd.0c00239

76. Lee DS, Sharabi M, Jefferson-Loveday R, Pickering SJ, Poliakoff M, George MW (2020) Scalable continuous vortex reactor for gram to kilo scale for UV and visible photochemistry. Org Process Res Dev 24:201-206. https://doi.org/10.1021/acs.oprd.9b00475

77. Harper KC, Moschetta EG, Bordawekar SV, Wittenberger SJ (2019) A laser driven flow chemistry platform for scaling photochemical reactions with visible light. ACS Cent Sci 5:109-115. https://doi.org/10.1021/acscentsci.8b00728 
78. Chaudhuri A, Kuijpers KPL, Hendrix RBJ, Shivaprasad P, Hacking JA, Emanuelsson EAC, Noël T, van der Schaaf J (2020) Process intensification of a photochemical oxidation reaction using a rotorstator spinning disk reactor: a strategy for scale up. Chem Eng J 400:125875. https://doi.org/10.1016/j.cej.2020.125875

79. Corcoran EB, Pirnot MT, Lin S, Dreher SD, DiRocco DA, Davies IW, Buchwald SL, MacMillan DWC (2016) Aryl amination using ligand-free Ni(II) salts and photoredox catalysis. Science 353:279. https://doi.org/10.1126/science.aag0209

80. Lévesque F, Di Maso MJ, Narsimhan K, Wismer MK, Naber JR (2020) Design of a Kilogram Scale, plug flow Photoreactor enabled by high power LEDs. Org Process Res Dev 24:2935-2940. https:// doi.org/10.1021/acs.oprd.0c00373

81. Corcoran EB, McMullen JP, Lévesque F, Wismer MK, Naber JR (2020) Photon equivalents as a parameter for scaling Photoredox reactions in flow: translation of Photocatalytic $\mathrm{C}-\mathrm{N}$ cross-coupling from lab scale to multikilogram scale. Angew Chem Int Ed 59: 11964-11968. https://doi.org/10.1002/anie.201915412

82. Saikia I, Borah AJ, Phukan P (2016) Use of bromine and Bromoorganic compounds in organic synthesis. Chem Rev 116:68377042. https://doi.org/10.1021/acs.chemrev.5b00400

83. Cantillo D, Kappe CO (2017) Halogenation of organic compounds using continuous flow and microreactor technology. React Chem Eng 2:7-19. https://doi.org/10.1039/C6RE00186F

84. Deshpande S, Gadilohar B, Shinde Y, Pinjari D, Pandit A, Shankarling G (2015) Energy efficient, clean and solvent free photochemical benzylic bromination using NBS in concentrated solar radiation (CSR). Sol Energy 113:332-339. https://doi.org/10.1016/ j.solener.2015.01.008

85. Eissen M, Lenoir D (2008) Electrophilic Bromination of alkenes: environmental, health and safety aspects of new alternative methods. Chem Eur J 14:9830-9841. https://doi.org/10.1002/ chem. 200800462

86. Steiner A, Williams JD, de Frutos O, Rincón JA, Mateos C, Kappe CO (2020) Continuous photochemical benzylic bromination using in situ generated $\mathrm{Br} 2$ : process intensification towards optimal PMI and throughput. Green Chem 22:448-454. https://doi.org/10.1039/ C9GC03662H

Publisher's note Springer Nature remains neutral with regard to jurisdictional claims in published maps and institutional affiliations.

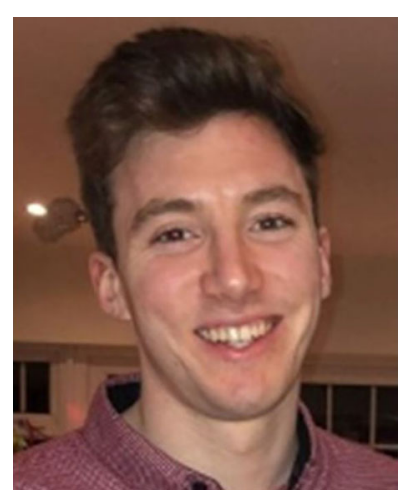

Kian Donnelly graduated from University College Dublin in 2019 with a $1^{\text {st }}$ class honours degree in chemistry. His final year project focused on applications of flow chemistry and he subsequently began his $\mathrm{PhD}$ with the Baumann group in 2019. His current research focuses on the continuous flow synthesis of strained cyclic molecules.

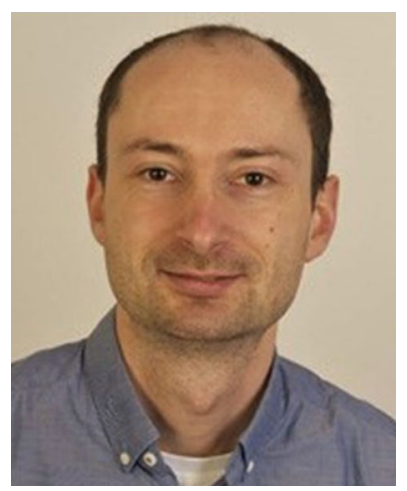

Marcus Baumann graduated from Philipps-University Marburg (Germany) in 2007 before moving to Cambridge to study for a PhD with Prof. Steven V. Ley FRS in the area of continuous flow synthesis. Subsequent postdoctoral studies with Prof. Larry E. Overman at the University of California in Irvine, and Prof. Ian R. Baxendale at the University of Durham followed.

In 2017 he joined the School of Chemistry at University

College Dublin as an Assistant Professor for Continuous Flow Chemistry, where his group's efforts centre around the development of new continuous flow methods applied to the effective generation of various target molecules. Current areas of interest include process development, reaction scale-up, photochemistry, telescoped multi-step sequences and biocatalysis. 